\title{
Salt Crystal Growth in Interacting Drops of a Complex Biopolymer: Statistical Characterization Using FESEM Images
}

Moutushi Dutta Choudhury ${ }^{1,3}$, Saptarshi Das ${ }^{2}$, Biswajit Roy ${ }^{1}$, Subhamita Sengupta $^{1}$, Sujata $_{\text {Tarafdar }}{ }^{1}$, and Sanat Karmakar ${ }^{1}$

1) Condensed Matter Physics Research Centre, Department of Physics, Jadavpur University, Kolkata-700032, India

2) Department of Mathematics, College of Engineering, Mathematics and Physical Sciences, University of Exeter, Penryn Campus, Penryn TR10 9FE, United Kingdom

3) Indian Institute of Science Education and Research (IISER) Mohali, Mohali, Punjab, India

\author{
Author's Email: \\ saptarshi@pe.jusl.ac.in, s.das3@exeter.ac.uk (S. Das) \\ roy.biswajit.99@gmail.com (B. Roy) \\ sengupta.subhamita@gmail.com (S. Sengupta) \\ sujata tarafdar@hotmail.com (S. Tarafdar) \\ sanatkarmakar@gmail.com (S. Karmakar)
}

moutushi@iisermohali.ac.in; mou15july@gmail.com (M. Dutta Choudhury)

\begin{abstract}
:
A simple colloidal drop generally forms ring like patterns after drying. The deposition morphology of the dried drop changes significantly when such a drop dries in the vicinity of another similar drop. Here we present an observational study and statistical analysis of the patterns formed inside an isolated as well as interacting drops of gelatin containing sodium sulfate $\left(\mathrm{Na}_{2} \mathrm{SO}_{4}\right)$. In all the cases, multiple concentric regions of solute particles combined with the polymer gel appear as the drops dry up. Needle crystals of sulfur and coacervates of salt and gelatin are visible in some regions. The outer region becomes non-uniform, so does the size distribution of the needle crystals and coacervates. The non-uniformity increases with proximity of the drops. Here we propose a novel mechanism of growing patterns inside the single drop during drying and correlate that with the results obtained for interacting drops. This study and the proposed mechanism provide insights into the future studies of drying drops under different physical conditions. Further we explore the statistical characteristics of the single and interacting drops using the field emission scanning electron microscopy (FESEM) images. Next, we report fractal and image texture analyses along with object shape statistics of the drop FESEM images, under various experimental conditions. Several statistical hypothesis tests have been carried out to identify the most significant features.
\end{abstract}

\section{Keywords:}

drop-drying, pattern formation, crystal growth, interacting drops, FESEM features, fractal analysis, image texture analysis

\section{Introduction}


Study of pattern formation in a drying liquid drop deposited on a solid substrate is an interesting and contemporary topic in fluid flow (Tarasevich et al. 2016; Deegan et al. 1997). A typical drop containing suspension of particles always leaves a ring of dirt deposited along the three-phase contact line (TPCL) after complete drying. This phenomenon is known as the 'coffee-ring' effect. The coffeering pattern arises when the drop is pinned on the substrate and maintains capillary flow during evaporation (Deegan et al. 1997)(Sefiane 2014). Another type of deposition is also observed near the central region following the Marangoni flow, which arises from the surface tension gradient as a result of temperature or concentration gradient (Hu \& Larson 2006).

\subsection{Background and Motivation}

Quite revolutionary changes came to food industry when spray drying was introduced to encapsulate food ingredients. This procedure of enriching flavours or quality of the food products is used since 1930s (Huang et al. 2017)(Zhang et al. 2017). But as the dehydration of the drops is involved here, the membrane of polymers encapsulating the product may vary due to improper distances between the drops. People have used several optimisers to reach proper microencapsulations. But here we propose that this encapsulation method could be insignificant if inhomogeneity forms at the outer boundary which depends on the distance between the drops.

The drop consists of salt ions in the biopolymer sol. During the $1^{\text {st }}$ half of the drying, crystallization occurred in the sol phase, and then the crystal structures are modified during the polymerization (Choudhury et al. 2013). It has been already observed by many researchers humidity effects the crystal structures (Pradhan \& Panigrahi 2015), (Choudhury et al. 2015) and different kinds of crystal separations can be done by using this property of crystal formation. It is quite similar to the magnetic nanoparticles (MNPs) growth processes in the presence of polymers (Maity et al. 2011). These are widely investigated due to their potential of interacting properties in biomedical applications, such as drug delivery, cell separation, genetic engineering etc. (Haracz et al. 2015). Therefore, study of interacting drops may help in understanding their applications in these fields. Research of plasmonics (Tao et al. 2007)(Fan et al. 2010)(Nakanishi et al. 2009) and metamaterials involve self-assembly of nanoparticles in super-lattice to observe their collective properties (Yoon et al. 2014)(Lewandowski et al. 2015)(He et al. 2014)(Dong et al. 2010)(Jiao et al. 2015). The collective arrangements of nanoparticles can be controlled by varying relative humidity $(\mathrm{RH})$ or simply placing another drop of the same colloidal solution. The same method can also enhance spectroscopies and properties of solar cells (Alvarez-Puebla et al. 2011)(Wang et al. 2007)(Rozin et al. 2015)(Zijlstra et al. 2009).

\subsection{Novelty of the present work}

The study of interacting drops has drawn a large number of applications such as DNA mapping (Jing et al. 1998), medical diagnosis (Brutin et al. 2011)(Yakhno 2008), drug delivery (He et al. 2003)(Wong 2011), inkjet printing (Park \& Moon 2006)(Layani et al. 2009) etc. Deposition of a complex fluid drop on a solid surface and letting it dry creates interesting patterns such as fractals (Choudhury et al. 2013), concentric rings (Choudhury et al. 2016)(Sefiane 2014), cracks (Khatun et al. 2013) etc. Interestingly, two identical drops of complex fluid, when placed closely, affect the deposition pattern of each other, after drying as reported in (Pradhan \& Panigrahi 2015; Pradhan \& Panigrahi 2016),(Edwards et al. 2015). It has been reported that the deposition patterns of drops of ink and water containing $1 \mu \mathrm{m}$ polystyrene particles change with the separation distance between the drops as the evaporation is slower from the nearest region than that from the other regions of the two drops (Pradhan \& Panigrahi 2015). This phenomenon is not only used to vary the deposition pattern but is also important in preparing different shapes of the residue of a colloidal drop. Formation of 
arched structures of the residue of colloidal alumina on alumina pillars was reported in (Chen \& Evans 2009) when the drops are allowed to dry adjacently. The proposed explanation behind this phenomenon is the effect of particle convection inside the drop due to non-uniform evaporation from the surface of the adjacent drops (Pradhan \& Panigrahi 2016)(Edwards et al. 2015). The coffee-ring effect of a single dried drop leads to particle separation which is used, reportedly, in nanochromatography of biomolecules (Wong et al. 2011).

In this context, the study of pattern formation in drying interacting drops of biological relevance can be used as a novel method for medical diagnosis and forensic science. In this work, we have studied the pattern formation in a drying drop composed of gelatin-sodium sulfate in the presence of a neighbouring drop. We propose a novel mechanism based on the kinetics during the evaporation and morphology of the dried up single drops, which is again correlated with changes found in case of interacting drops.

\section{Experimental Methods}

\subsection{Preparation of Gelatin Solution Containing $\mathrm{Na}_{2} \mathrm{SO}_{4}$}

For the experiments, sodium sulfate $\left(\mathrm{Na}_{2} \mathrm{SO}_{4}\right)$ is obtained from Merck (Mumbai, India) and gelatin from Lobachemie (Mumbai, India). In the beginning, we prepare $3 \mathrm{wt} \%$ of $\mathrm{Na}_{2} \mathrm{SO}_{4}$ water solution. Now $0.5 \mathrm{~g}$ gelatin was added to the formerly prepared $\mathrm{Na}_{2} \mathrm{SO}_{4}$ water solution and stirred in a magnetic stirrer at $60^{\circ} \mathrm{C}$ in order to make homogeneous solution of final concentration $1 \mathrm{wt} \%$. The solution is then allowed to cool at room temperature $\left(25^{\circ} \mathrm{C}\right)$ before starting the experiment.

\subsection{Deposition of Drops}

The optically smooth microscopic glass slides are cleaned with Extran detergent (Merck, Mumbai), then rinsed with de-ionized water and ethanol repeatedly to remove any impurity. Initially single drop of $\mathrm{Na}_{2} \mathrm{SO}_{4}$ water solution is deposited on one glass slide. Simultaneously on a similar glass slide an isolated drop of $\mathrm{Na}_{2} \mathrm{SO}_{4}$ gelatin solution is deposited.

In another set of experiment, two drops of $\mathrm{Na}_{2} \mathrm{SO}_{4}$ gelatin solution are deposited alongside. The distance between edge to edge of the two drops varies from $100 \mu \mathrm{m}$ to $700 \mu \mathrm{m}$. In all cases volume of the single drop is taken as $5 \mu \mathrm{l}$. All the drops are allowed to evaporate at relative humidity $53 \pm 2 \%$ and temperature $25^{\circ} \mathrm{C}$. We also vary relative humidity from $46 \pm 2 \%$ to $86 \pm 2 \%$ by keeping the drops in a closed chamber containing saturated solution of Potassium Carbonate $\left(\mathrm{K}_{2} \mathrm{CO}_{3}\right)$ and Potassium Chloride $(\mathrm{KCl})$. We check the reproducibility of the patterns under corresponding ambient conditions.

\subsection{Imaging of the Pattern Formation of Drying Drops}

The patterns in the dried drops are characterized by three different techniques. Low-resolution images are photographed using Nikon CoolPix L120 camera. Intermediate resolution images are obtained using an optical microscope (Leica DM 750) and high resolution images are obtained from FESEM (FEIC-QUO-35357-0614 with Bruker Quantax 100).

\subsection{Wettability Pattern}

Time evolution of contact angle, base diameter, height and volume of drops are measured by automated optical contact angle (OCA) goniometer and the data is analysed using SCA 20 (DataPhysics Instruments, $\mathrm{GmbH}$ ) software. The circle is fitted to drop-shape and various parameters are estimated using spherical cap model. Data are recorded for drying of the single drop as well as double drops. 


\section{Results for Single Drop}

\subsection{Pattern Formation in Drying Drop of $\mathrm{Na}_{2} \mathrm{SO}_{4}$ Solution}

We observe dendritic growth inside and creeping of salt crystals outside the drying drop of $\mathrm{Na}_{2} \mathrm{SO}_{4}$ solution, as shown in Figure 1. The solute particles forming a ring like pattern (coffee-ring effect) contouring the dried-up drop can be observed in Figure 1(a). The creeping of the dendritic salt crystals outside the boundary line and towards the centre could be observed clearly from the microscopic view of a part of the aforementioned ring Figure 1(b). Creeping salt crystals of $\mathrm{Na}_{2} \mathrm{SO}_{4}$ is discussed briefly in the work of Bonn et al. (Shahidzadeh-Bonn et al. 2008).

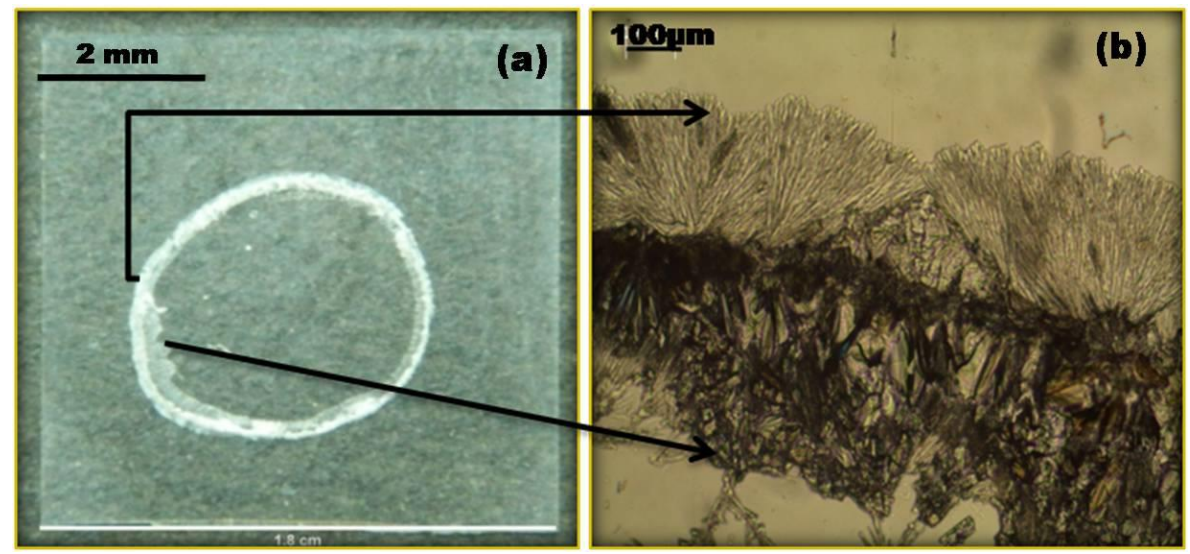

Figure 1: (a) Dried drop of $\mathrm{Na}_{2} \mathrm{SO}_{4}$ solution, (b) Creeping of salt crystal outside the drop as well as dendritic growth inside.

\subsection{Pattern Formation of $\mathrm{Na}_{2} \mathrm{SO}_{4}$ Solution Drop Containing Gelatin}

The patterns in dried drop of $\mathrm{Na}_{2} \mathrm{SO}_{4}$ containing gelatin show four distinct regions in Figure 2. The widths of the first and second regions are $\sim 25 \mu \mathrm{m}$ and $\sim 10 \mu \mathrm{m}$ respectively. The first region consists of large number of needle-like crystals of $\mathrm{Na}_{2} \mathrm{SO}_{4}$ in Figure 2(a) whereas the second region consists of a few needle crystals and their aggregates in Figure 2(b).
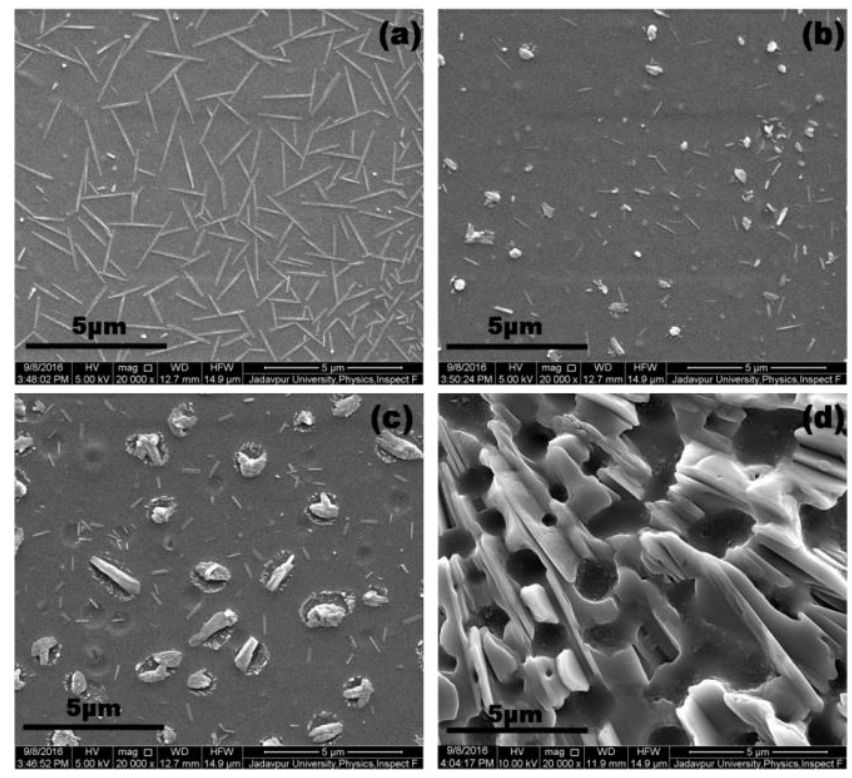

Figure 2: A dried drop of gelatin containing $\mathrm{Na}_{2} \mathrm{SO}_{4}$ salt consists of four regions: (a) outer most needle-crystal region, (b) intermediate region of needle-crystals and its aggregates, (c) aggregates of the needle crystals, (d) central region. 
The aggregates region (width $\sim 50 \mu \mathrm{m}$ ), consists only of the aggregates of needle crystals Figure 2(c). In this region, the sizes of the aggregates of needle crystallites are found to be larger than that in the intermediate region. EDX results in Figure 3(a) confirm that the background of these aggregates contains gelatin. At the central region of the dried drop floral pattern of $\mathrm{Na}_{2} \mathrm{SO}_{4}$ in the presence of gelatin is noticed, as shown in Figure 3(b). We believe that the salts accumulate in this region leading to the observed floral pattern.
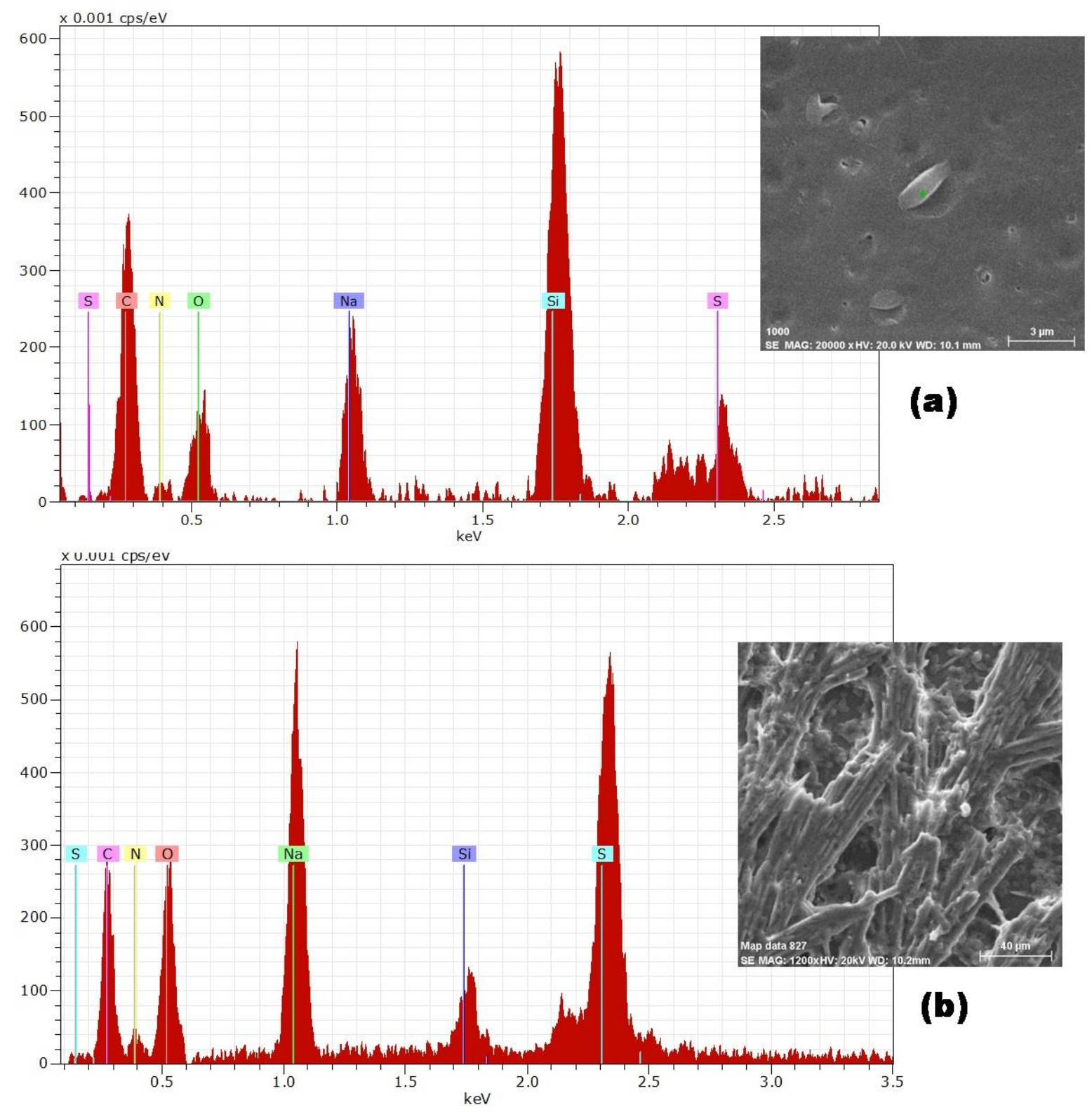

Figure 3: (a) EDX spectra of the aggregates of the needle crystals and (b) EDX spectra of the central region.

\subsection{Mechanism of Pattern Formation of a Single Drying Drop}

We believe that the formation of these nontrivial patterns in a single drop of this complex fluid might be the consequence of the Marangoni effect. It is well known that when a drop of aqueous solution is deposited on a partially hydrophilic substrate, the evaporation flux, from the drop surface is non-uniform and is maximum along the TPCL and minimum at the apex of the drop (Pradhan \& 
Panigrahi 2015)(Deegan et al. 1997). It diverges as the instantaneous radius of a drop approaches the three phase contact line as:

$$
J_{s} \sim(R-r)^{\lambda}
$$

and

$$
\lambda=\frac{\pi-2 \theta_{c}}{2 \pi-2 \theta_{c}},
$$

where, $R$ is the initial radius of the drop and $\theta_{c}$ is the angle subtended by the deposited drop with the substrate.

Accordingly, the 3-phase contact line tends to cool faster than the other regions of the drop resulting in an increase in the surface tension, along the TPCL. But the substrate is still now hotter than the drop because of evaporative cooling and consequently, there would be a temperature gradient developed between the substrate and the drop, as shown in Figure 4.

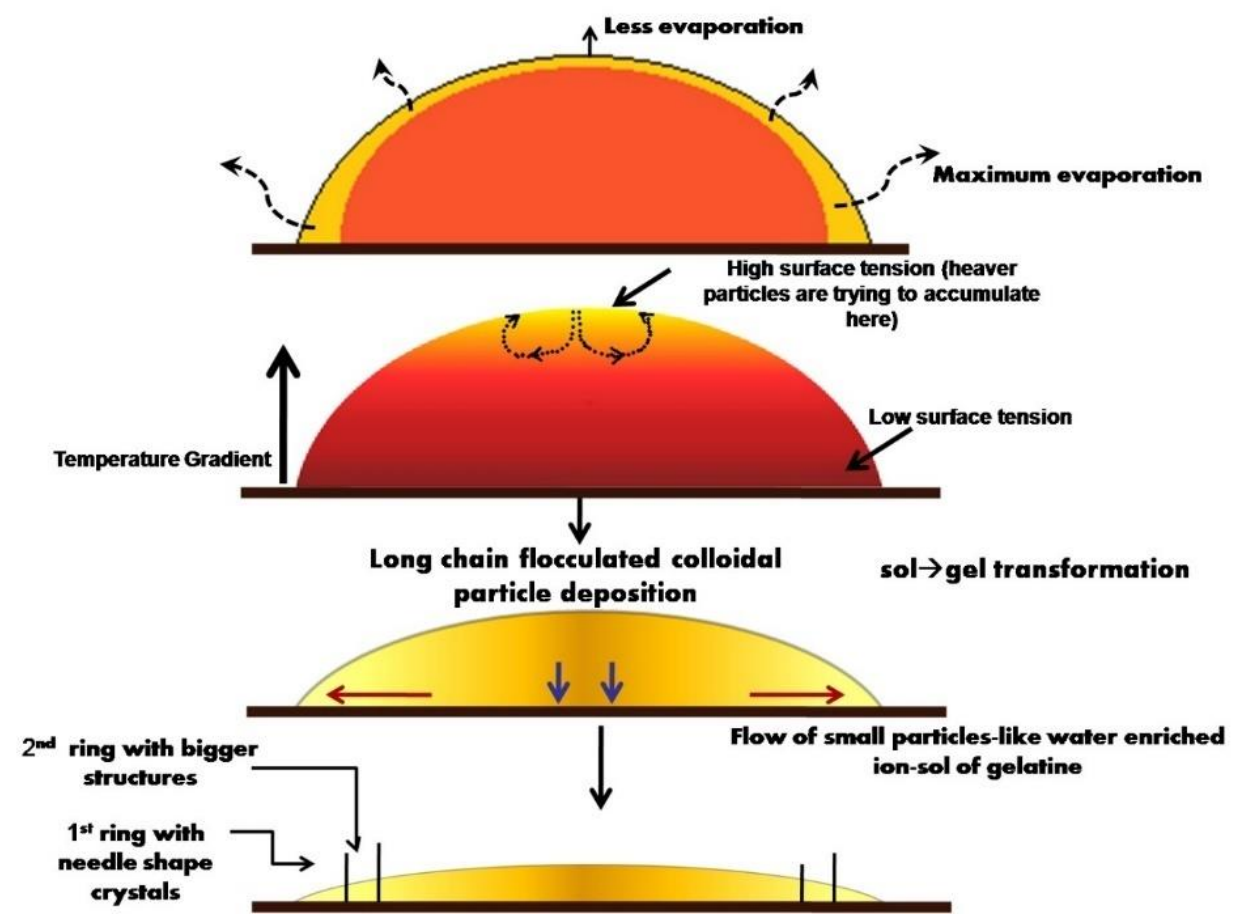

Figure 4: In the schematic diagram of a single $\mathrm{Na}_{2} \mathrm{SO}_{4}$ gelatin drop-drying mechanism, cooling is represented by yellow colour, whereas hotter regions are represented by darker reddish colours.

The temperature gradient throughout the drop generates a surface tension gradient and Marangoni flow takes place (Hu \& Larson 2006). With time, sol-gel transition ensues and the heavier flocculated colloidal gel-salt tends to deposit at the centre of the drop. The surface tension force is more dominant at this point compared to both gravitational pull (since the size is less than capillary length) and viscous force (since the major portion of the drop is still in sol phase).

In the mean-time, the height $(H)$ of the drop decreases, keeping three phase contact line pinned, resulting in capillary flow of lighter particles, outward from the centre. At this stage Marangoni flow ceases to exist due to the significant decrease in the Marangoni number $\left(M_{a}\right)$ to a critical value. It is clearly evident from equation (3) that this can happen due to increase in viscosity $(\mu)$ and thermal diffusivity $(\kappa)$ in the presence of gelatine. Marangoni number $\left(M_{a}\right)$ is given by: 


$$
M_{a}=\frac{\partial \gamma}{\partial T} \frac{H L \Delta T}{\mu \kappa}
$$

The $M_{a}$ decreased to a critical value as, viscosity $(\mu)$, increases hugely (values of viscosity change from $160 \mathrm{mPa}$ s to $1000 \mathrm{mPa}$ s) and so does the thermal diffusivity $\kappa$, because of gelation.

Since the drop becomes very thin at this stage, temperature gradient decreases and there is increase in $\kappa$. The $\mu$ and $\kappa$ predominate over the surface tension $(\gamma)$ of the fluid. Therefore, particles, which cannot move inward from the outer layer, form needle like crystals. The formation of first region is followed by a second region, as there is still enough gel and aqueous solvent left. The evaporation in the second region results in a coagulation of needle crystallites to larger structures, as shown in Figure 4.

The salt within the drop tries to retain its crystal growth conditions owing to its hygroscopic nature and during crystal formation, salt crystallites separate partially from the gel. Coacervates of gelatine and salt are formed with circular contours. It also appeared that the cracked and broken crusts on the large rounded deposits look like blisters, which form by fluid/vapour accumulating below a skin and subsequent swelling. Since these salt aggregates are encapsulated with gelatine the EDX spectra show a strong peak of carbon at this spot as in Figure 3(a). At the end of the drying process, the curvature of the drop remains same throughout the drop-surface. This leads to uniform evaporation of the drop which results at the middle section of the drop as shown in Figure 2(d).

We have performed the same experiment for single drop in a closed chamber at relative humidity $86 \%$ and the aggregated needle crystals with circular boundaries are spotted in Figure 5 which are more in number than that observed at humidity $46 \%$. The slow evaporation rate at high RH retains water in the drop for more time which is the reason for such accumulated crystal configuration. Needle shaped crystals of salt are observed more in low RH whereas very few number of needles are noticed in high RH.
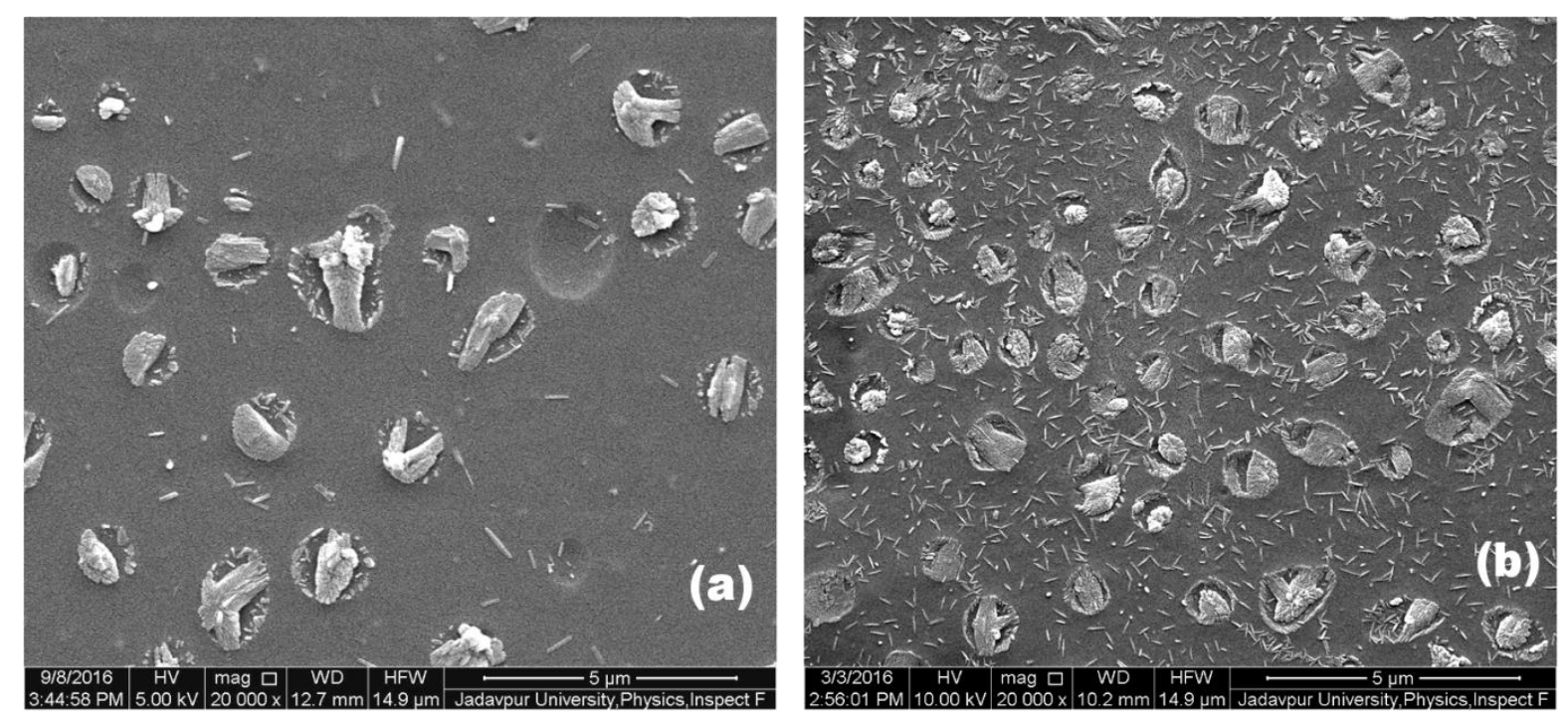

Figure 5: (a) Aggregates of the needle crystals of a single drop in humidity $86 \% \pm 5 \%$ and surrounding temperature $22^{\circ}$, (b) same region for single drop, but humidity is $46 \% \pm 5 \%$.

\section{Results for Interacting Drops}

The mechanism of pattern formation, as well as final patterns of the interacting drops are found to be quite different from that of an isolated single drop. For example, the width of the regions at the 
close proximity of the two drops is observed to be significantly smaller than that of a single drop Figure 6(a). In the highest proximity region, the number of single needle crystals is found to be negligible and the aggregates of needle crystals increase in size while the number of aggregates decrease. But the width of the same region in the non-interacting part of the drop is much higher than that of the proximity region. The needles of $\mathrm{Na}_{2} \mathrm{SO}_{4}$ are clearly visible in Figure $6(\mathrm{~b})$ and they are quite large in number, though very small in length. It is clear that the needles couldn't get much time to aggregate at this region and they are unable to form aggregation like Figure 6(a). The nature of the pattern at the central region of each of the double drops are almost the same as that at the centre of an isolated dried drop.

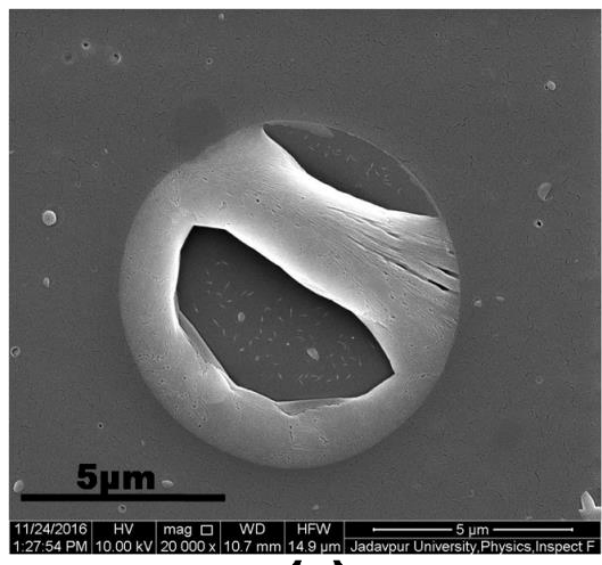

(a)

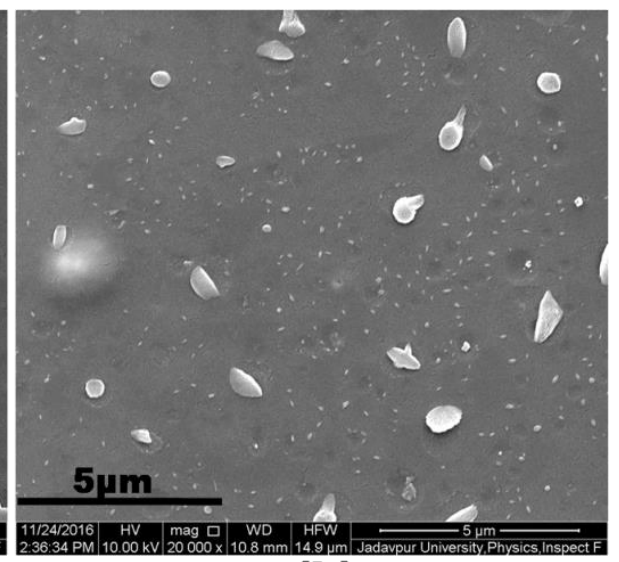

(b)

Figure 6: (a) Interacting region of a drop among two drops, (b) rear region of one of these two drops.

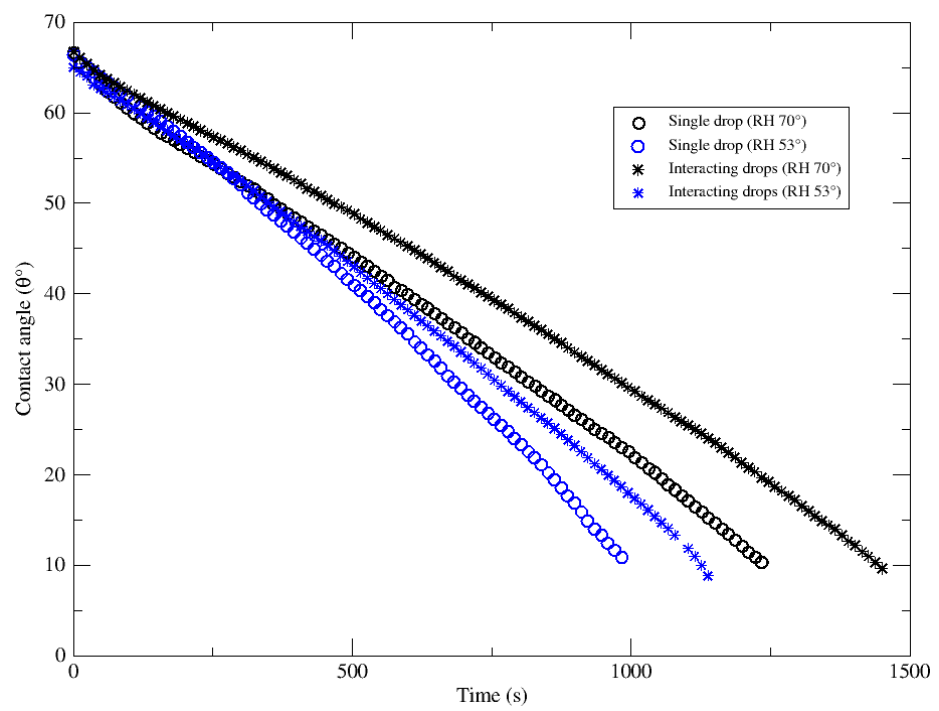

Figure 7: This figure depicts the mean contact angle variations with time for single drops (black and blue circles) and the interacting drops during drying (black and blue stars). Black symbols are for humidity $70 \pm 2^{\circ}$, blue symbols are for lesser humidity $\left(53 \pm 2^{\circ}\right)$.

We have also observed that when the gap between two drops is $\geq 350 \mu \mathrm{m}$, the structures inside the dried double drops are very similar to a dried single drop. The evaporation rate is different in double drops from the single drop as evident from the measurement of the contact angle with time. Interestingly, it is clearly evident from Figure 7 that, the contact angle is higher for interacting drops at the proximity region than for the single drop. The evaporation flux is not uniform at the TPCL for interacting drops, unlike the single drop. 
Region (b) (interacting region) of the Figure 8(A) contains more water vapour (vapour concentration $C_{a}$ ) than the region (a) and (c) (vapour concentration $C_{\infty}$ ) at the same time. Therefore, the diffusion of vapour from the surface (vapour concentration $C_{s}$ ) to ambient air is less at (b) in Figure 8(A). So, the crystals get more time to aggregate. It is similar to the case of a single drop drying in a closed chamber where the aggregates are bigger in size in the aggregated region. We have observed the widths of interacting and rear regions are uniform throughout the dried single drop but we have found different widths for the case of interacting drops.

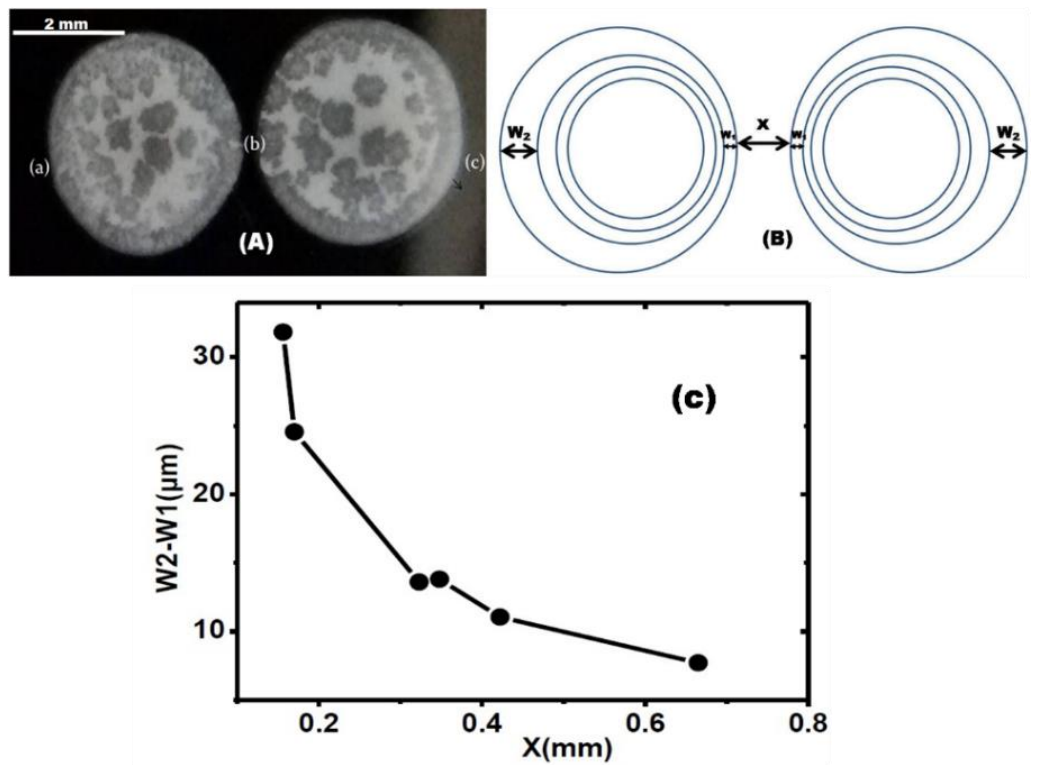

Figure 8: (A) Image of two interacting drops with separation distance $176 \mu \mathrm{m}$, (B) schematic diagram shows how the width of the outer regions changes with distance $x,(\mathrm{C})$ Variation of the difference between the widths of the outer most regions in the proximity part of the drops with the separation $\mathrm{x}$ between the two drops.

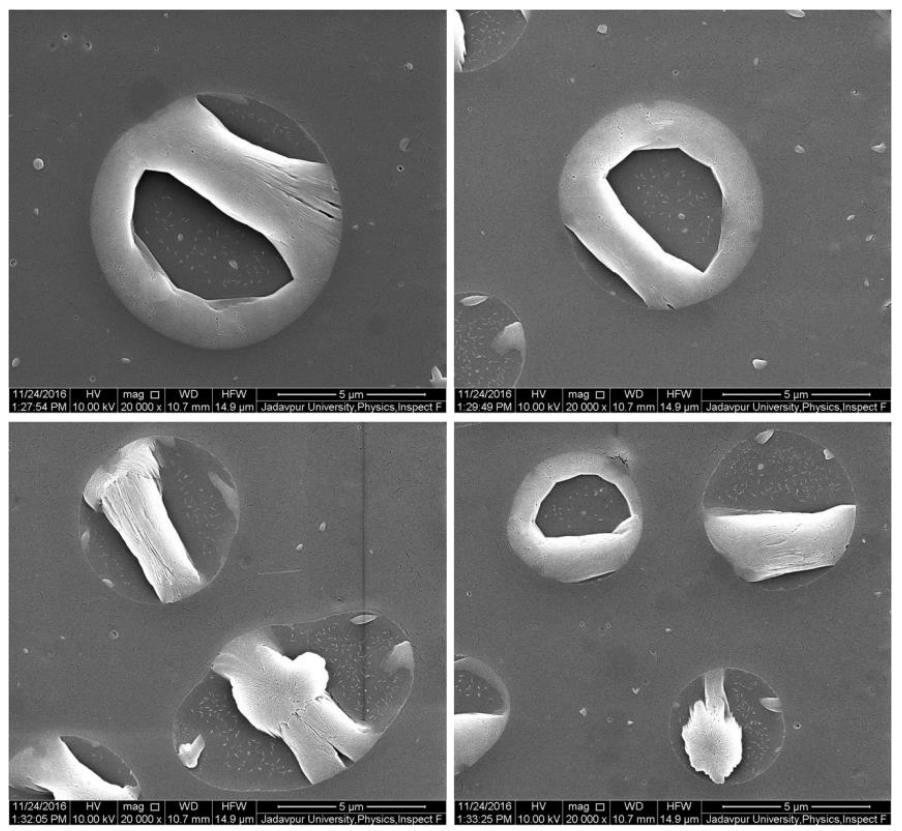

Figure 9: Broken blister like structures from the interacting part of the double drops. Needle crystal aggregations inside the broken blisters are also evident. However the four subpanels show same region of different drops. Variation of the size and shape of the blisters are noticed but the crystals inside seem to be similar which are analysed later using the SEM image statistics. 
The width of the $1^{\text {st }}$ and $2^{\text {nd }}$ regions at the point (b) is lower than that at the point (a) and (c) in the drops as shown in Figure 8. The outer regions are very thin and nearly transparent at the point (b). Figure $8(\mathrm{c})$ shows that the difference in width decreases with increasing separating distance $x<350$ $\mu \mathrm{m}$. As $x$ increases, i.e. the patterns appear similar to a single drop. This confirms the non-uniformity in the widths of the regions in the proximity part of the drops due to interaction. We tried to control the spacing between the drops by depositing more than 50 pairs of drops manually and selecting those pairs of drops which were separated by the distance less than $350 \mu \mathrm{m}$. Since the drop spreads after deposition the distance between edges of the drops could not be controlled. Here we made an effort to find the relation between theses spacing between the drops with the width of outer ring of the drops as shown in Figure 8. The width of the ring increases with decreasing distance and it also controls the salt flocculation at the edge. Basically the spacing is the key factor for controlling the humidity between the regions of two drops. It was reported in (Pradhan \& Panigrahi 2015) that diffusion of the vapour from the outermost surface of the drops during evaporation reduces if the space between the drops are reduced.

We have come across interesting features in dried interacting drops when the relative humidity varies from $46 \%$ to $86 \%$ at temperature $~ 25^{\circ} \mathrm{C}$ as in Figure 5. When relative humidity is quite high (86\%) needle shaped crystals get more time to accumulate, but the underlying mechanism is very different when humidity is low (46\%) enough. During fast drying i.e. at low $\mathrm{RH}$, the needle crystals (the primary structure of $\mathrm{Na}_{2} \mathrm{SO}_{4}$ salt) do not get time to combine and most of them are left isolated (Choudhury et al. 2016). Even in this case, the small needles could be noticed inside the circular parts in salt aggregated region. For slow drying process, i.e. at high RH, larger aggregates are noticed. These observations fully support our proposed mechanism of formation of larger aggregates of needle crystals in the nearer periphery of the drop in the presence of another drop.

It is apparent that the evaporation occurred at the base edge of the drop. The fluid flows towards the Three Phase Contact Line (TPCL) to maintain the constant contact radius (CCR) mode. But during this time, the liquid is a sol of gelatin and salt. It was observed earlier in (Shahidzadeh-Bonn et al. 2008) that needle like crystal is the basic structure of $\mathrm{Na}_{2} \mathrm{SO}_{4}$. But fast evaporation leads oversaturated $\mathrm{Na}_{2} \mathrm{SO}_{4}$ at the periphery and we observe only needle structures at the outermost region $(\sim 100 \mu \mathrm{m})$. Since $\mathrm{Na}_{2} \mathrm{SO}_{4}$ salt drop creates prominently mesoscopic film at the periphery of a drop, the presence of another drop plays an important role in the formation of crystals at the nearer end of the two drops, as shown in Figure 6 and Figure 9. Sol-gel transformation occurs after formation of the $1^{\text {st }}$ outer thin region. Gel transformation changes the viscosity of the fluid remarkably (Choudhury et al. 2013), (Dutta et al. 2013). That is why the latter regions take more time to dry. Also at the middle, we found more occurrences of gelatin influenced salt aggregations. Most of the gel containing gelatin and $\mathrm{Na}_{2} \mathrm{SO}_{4}$ congregates at the centre of the drop because of its convex shape as shown in the EDX spectra in Figure 3.

It was found that some layers reside on the slide if we wash it lightly after allowing the drop to dry for 2-3minutes. These micro films of the dried liquid contain gelatin as well as small quantity of salt (Dutta et al. 2013)(Choudhury et al. 2015). The process of mixing salt in the polymer gel or the formation of polymer sol in saltwater decides the 'crystal' amorphous structures. Several concentric rings are observed for preceding case (Roy et al. 2015), no large deposition at the centre was observed. But in the latter case, we observed quite different aggregations. It has been established in (Choudhury et al. 2013)(Choudhury et al. 2015)(Zang et al. 2019) that the aggregation changes with changing polymers in the droplet. Also EDAX spectra in Figure 3 confirm the presence of gelatin on the solid aggregates. 
We have also investigated the effects of $\mathrm{pH}$ of the solutions and salt type on the pattern formation of drying drops. The $\mathrm{pH}$ of the solutions were varied as $6.47,7.18,8.60$ and 9.27. We did not find any significant change in the pattern of the dried single and interacting drops at these $\mathrm{pH}$. However, we indeed find a significant change in the patterns for some alkali metal salts, such as, such as $\mathrm{NaCl}$, $\mathrm{KCl}, \mathrm{NaBr}, \mathrm{KBr}, \mathrm{NaI}$, KI. Unlike the pattern formation described in the present study, faceted crystals and dendritic crystals in the dried drops of these salt-gelatin solutions have been observed, but they do not interact significantly in presence of another drop. The mechanism of the crystal growths of $\mathrm{NaCl}$ with varying RH have been explained qualitatively in earlier literatures (Choudhury et al. 2016; Choudhury et al. 2013). In the $\mathrm{Na}_{2} \mathrm{SO}_{4}$ gelatin solution, the sulfur plays a crucial role in the process of pattern formation. It is reported that for salting out of hydrophilic colloids, like gelatin, from watery solution, sulphates are more efficient than chlorides regardless the $\mathrm{pH}$ of the gelatin solution (Loeb \& Loeb 1921).

\section{Statistical Analysis of the FESEM Images}

Next using the FESEM images of the single and interacting drops, we now quantify the experimental pattern formation in terms of few statistical features. It was made sure that the magnification factors of the FESEM images were the same as 20,000x for analysing all the patterns at the same scale. The statistical analysis reported in this section has been inspired from earlier FESEM image analysis methods proposed in (Dutta Sinha et al. 2017). Here we mainly report three types of statistical analysis viz. image texture, fractal and statistics of the number and shape of the objects in an FESEM image. Using these measures, we then carry out several hypothesis tests to find out the similarity and difference between these patterns which is quantifiable via the most significant features.

\subsection{Texture Analysis}

First, we calculate textural features from each FESEM images for different areas of the drops and under different conditions. This includes first and second order statistics of the pixel intensities. The first order statistics essentially capture various aspects of the pixel intensity histograms as shown in Figure 10 for the different parts of single drop, then single drop with humidity effects and for the interacting drops. The pixel intensities of a FESEM image can be used to calculate the shape parameters or higher moments of the underlying distributions e.g. mean $(\mu)$, standard deviation $(\sigma)$, skewness $(\gamma)$, kurtosis $(\beta)$, energy $(E)$, entropy $(H)$, smoothness texture (SM) etc. A more skewed distribution is observed in the central region of Figure 10(a) and for the interacting drops in Figure 10(c) indicating the abundance of brighter pixels than darker ones. All these features together characterise the pattern formation due to drop drying under various scenario. Next, we also calculate the second order statistics of the images which capture the spatial correlation structure of the FESEM images. These features of second order statistics include - contrast, spatial correlation, homogeneity, uniformity or 2D energy. These feature scan be calculated from the gray level co-occurrence matrix (GLCM) and have been thoroughly described in (Dutta Sinha et al. 2017)(Haralick et al. 1973). 

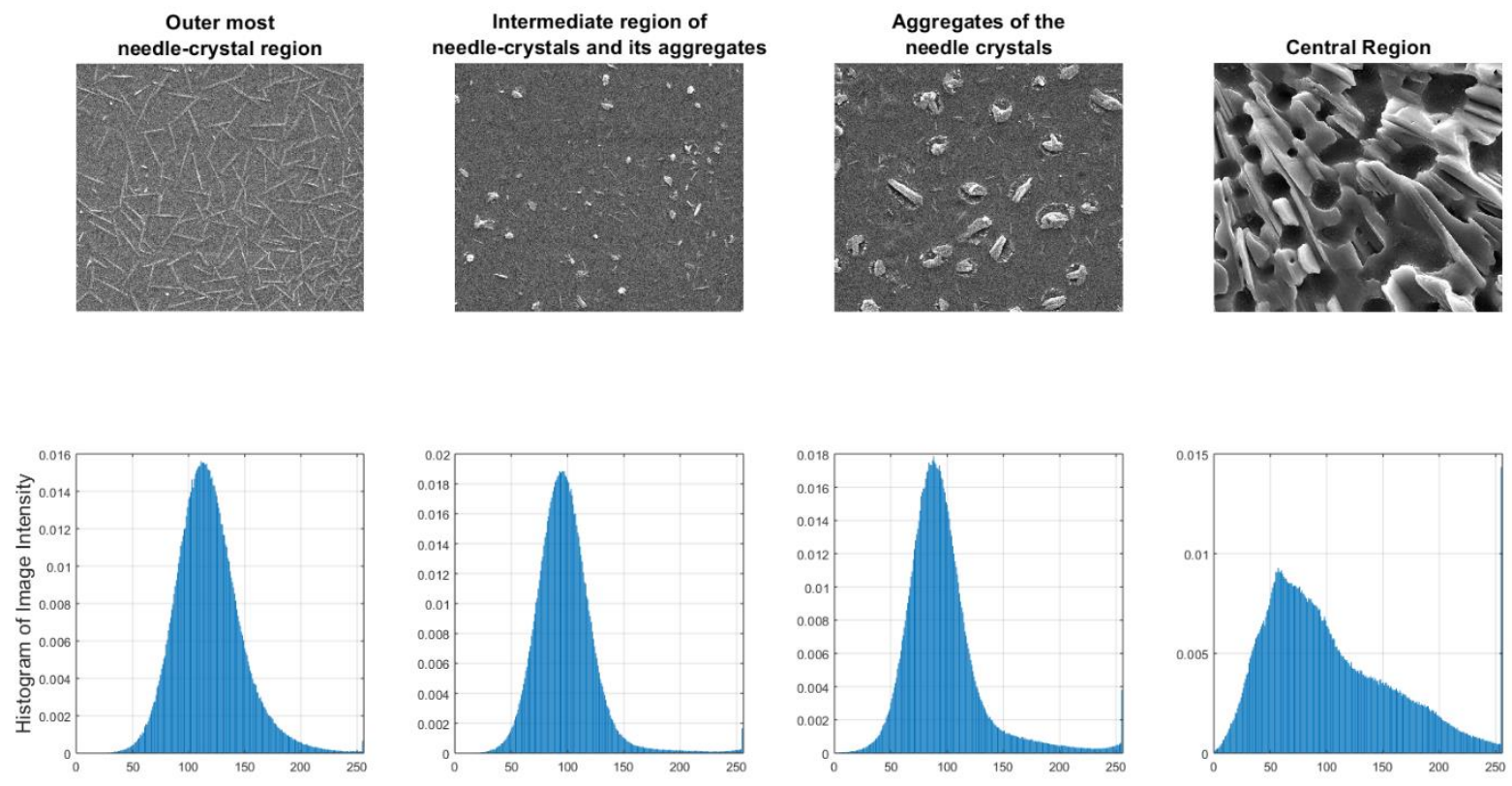

(a)
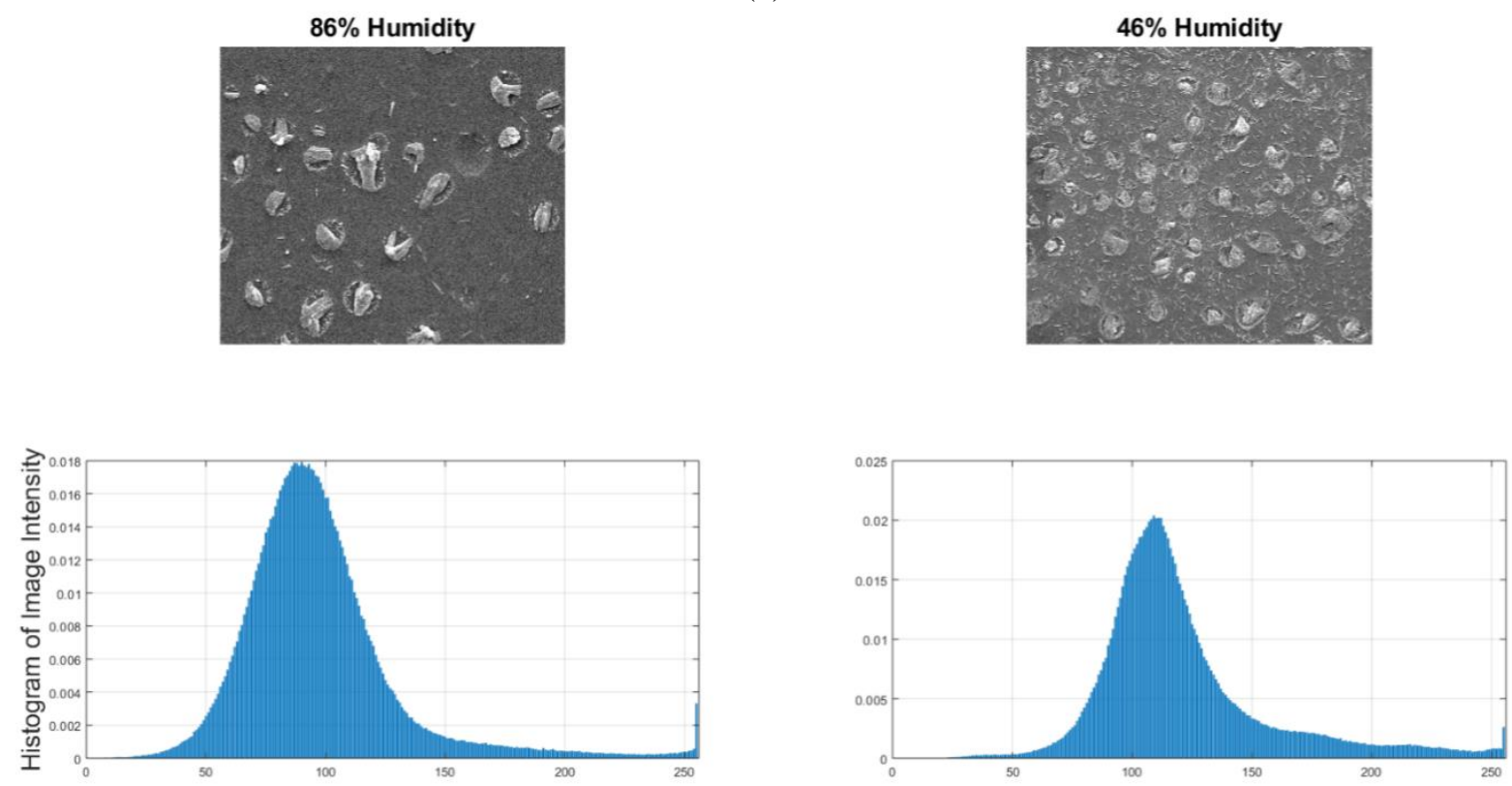

(b) 

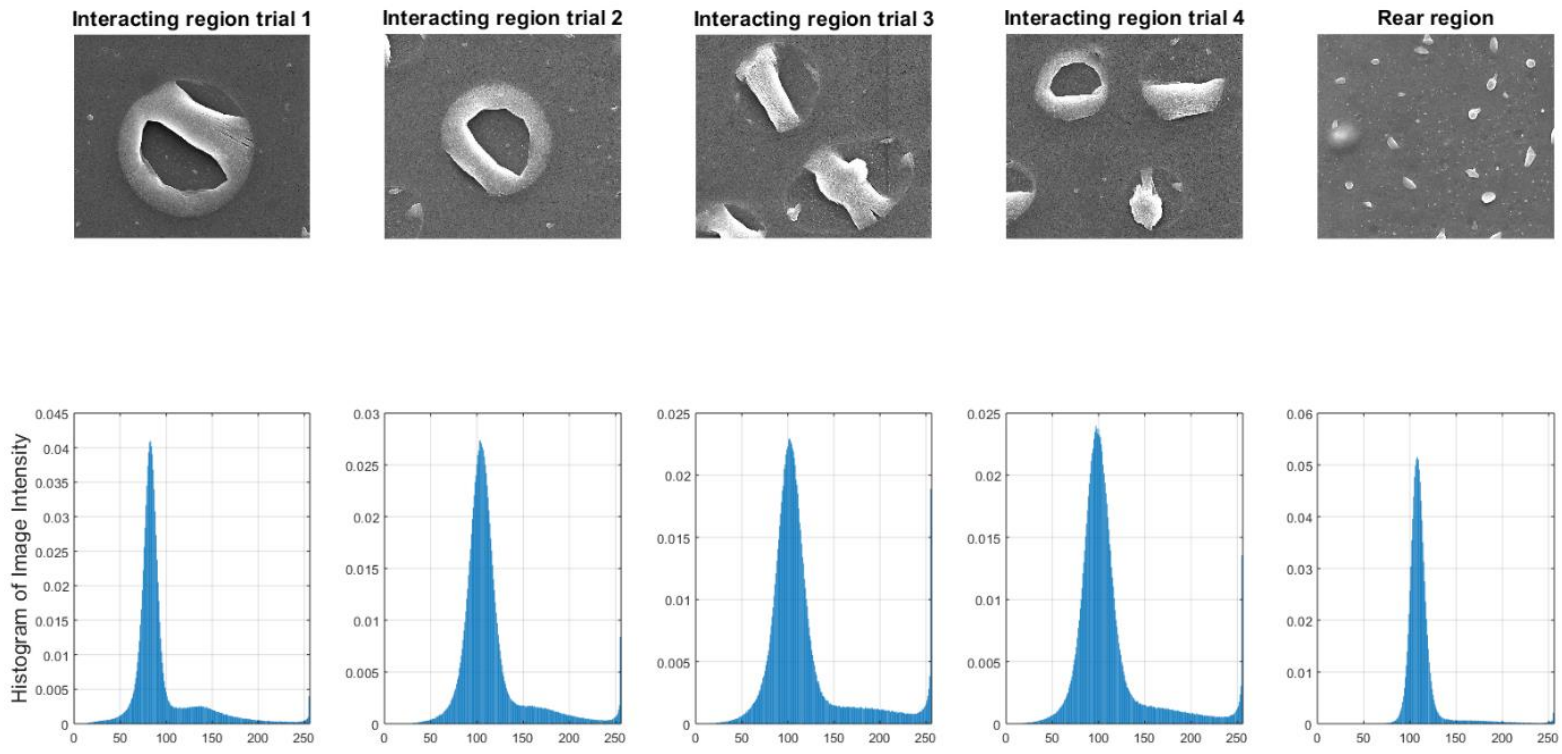

(c)

Figure 10: Intensity histograms for (a) single drop different parts, (b) single drop with humidity effects, (c) interacting drop. The $x$-axis of the histograms represent the pixel intensity and the $y$-axis represent the normalized frequency of occurrence for different pixel intensities. These figures show the grayscale images of single and interacting drops for different trials and regions. It also shows the distribution of the pixel intensities for these various cases where heavy tailed and skewed distributions are found. This helps in quantitative characterization of these images by extracting various statistical features of these pixel intensity histograms to allow comparison of various conditions.

\subsection{Fractal Analysis}

Next, we apply the fractal analysis method using box-counting to calculate the Hausdorff or fractal dimension $\left(D_{f}\right)$ of the images. First the grayscale FESEM images have been binarized using threshodling methods e.g. Otsu's method (Otsu 1979). However, other studies have also suggested that an adaptive thresholding (Bradley \& Roth 2007) or pixel intensity clustering-based methods (Arifin \& Asano 2006; Pappas 1992) can also be used for this purpose. Using the converted binary images with only white and black pixels, now the number of black pixels are counted with increasing box size. This method yields the box-counting results plotted on a log-log graph of box size vs. number of black pixels. For each binary image's box-counting data, a straight line was fitted whose slope can be used to estimate of the fractal dimension of the image. This method has been described in a greater detail in (Feder 2013; Tamas 1992). Apart from calculating, $D_{f}$ we also report the coefficient of variation $\left(R^{2}\right)$ values for each estimates of fractal dimension that indicate how closely the data points lie on a straight-line in the log-log graphs and are shown in Figure 11 for three cases of pattern formation due to drop drying. Previous similar experimental fractal pattern formation can be viewed in the context of viscous fingering and crack formation during drying of complex fluids in (Sinha \& Tarafdar 2009; Tarafdar \& Sinha 2008; Roy et al. 1999; Mal et al. 2006). In Figure 11, it is to be noted that the two thresholding methods have been used primarily for converting the grayscale images to the respective binary ones for fractal investigations which should not be directly interpreted to salt depositions in the white pixel areas. It is also evident that the Otsu's thresholding method smears out many finer structures in the scene which makes it less robust for fractal dimension calculation which also gets reflected from a relatively lower $R^{2}$ value compared to the adaptive thresholding method. 
One requirement while binarizing a grayscale image is that it should retain the fine structures in the scene in the binary version as close as possible to the original grayscale image. To explore this more we have compared the binary image conversion step using two different algorithms - Otsu's method (Otsu 1979) and adaptive thresholding method (Bradley \& Roth 2007). The respective fitting results for fractal analysis using the box-counting methods are shown in Figure 11 for the three cases of drop drying patterns. It is apparent from Figure 11 that the adaptive thresholding method is capable of retaining the finer structures of the converted binary image similar to the original grayscale image. Therefore, in the hypothesis testing reported in the next section, only the features calculated using this particular thresholding method have been used and not the features using Otsu's method.
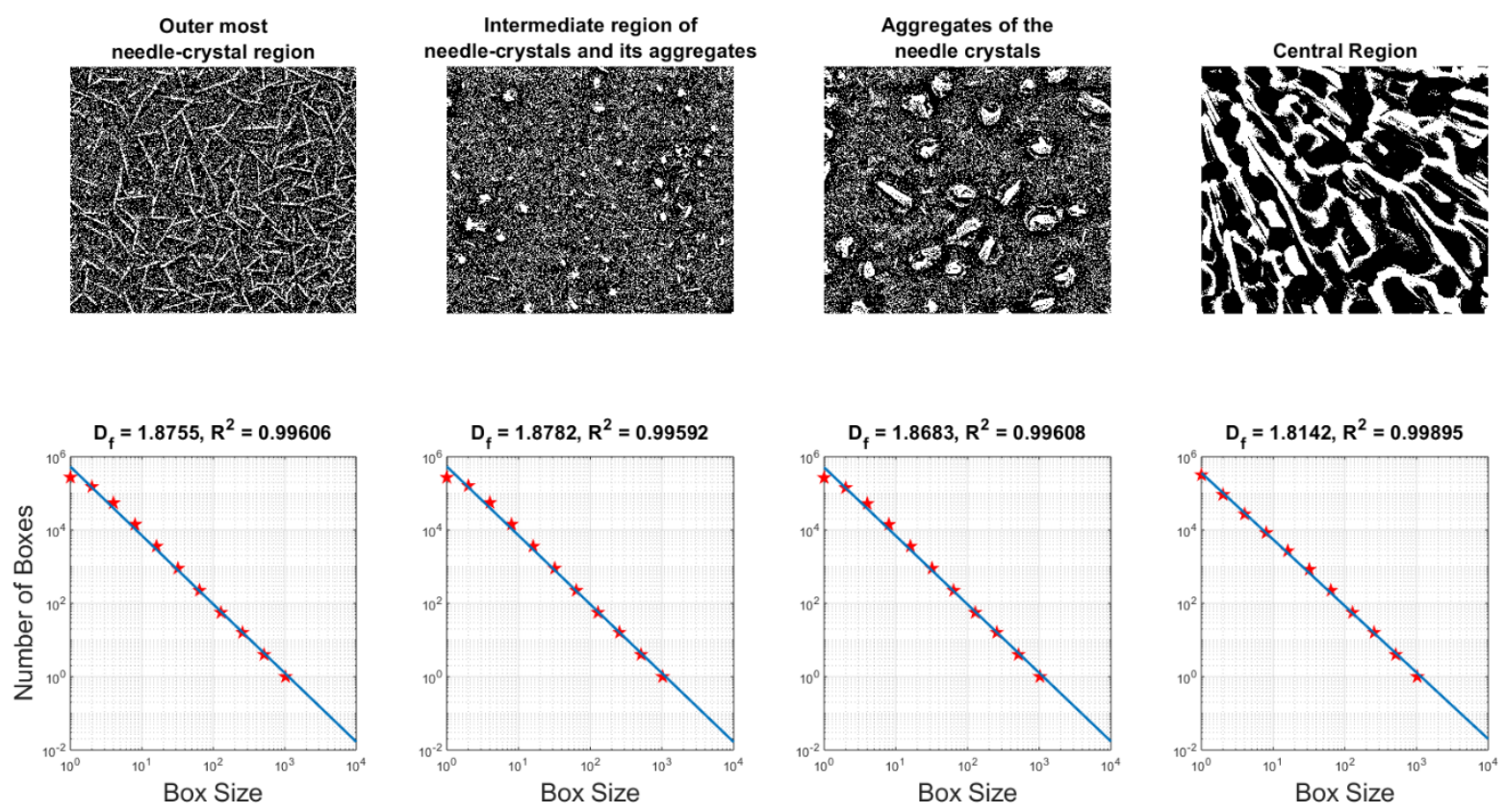

(a)
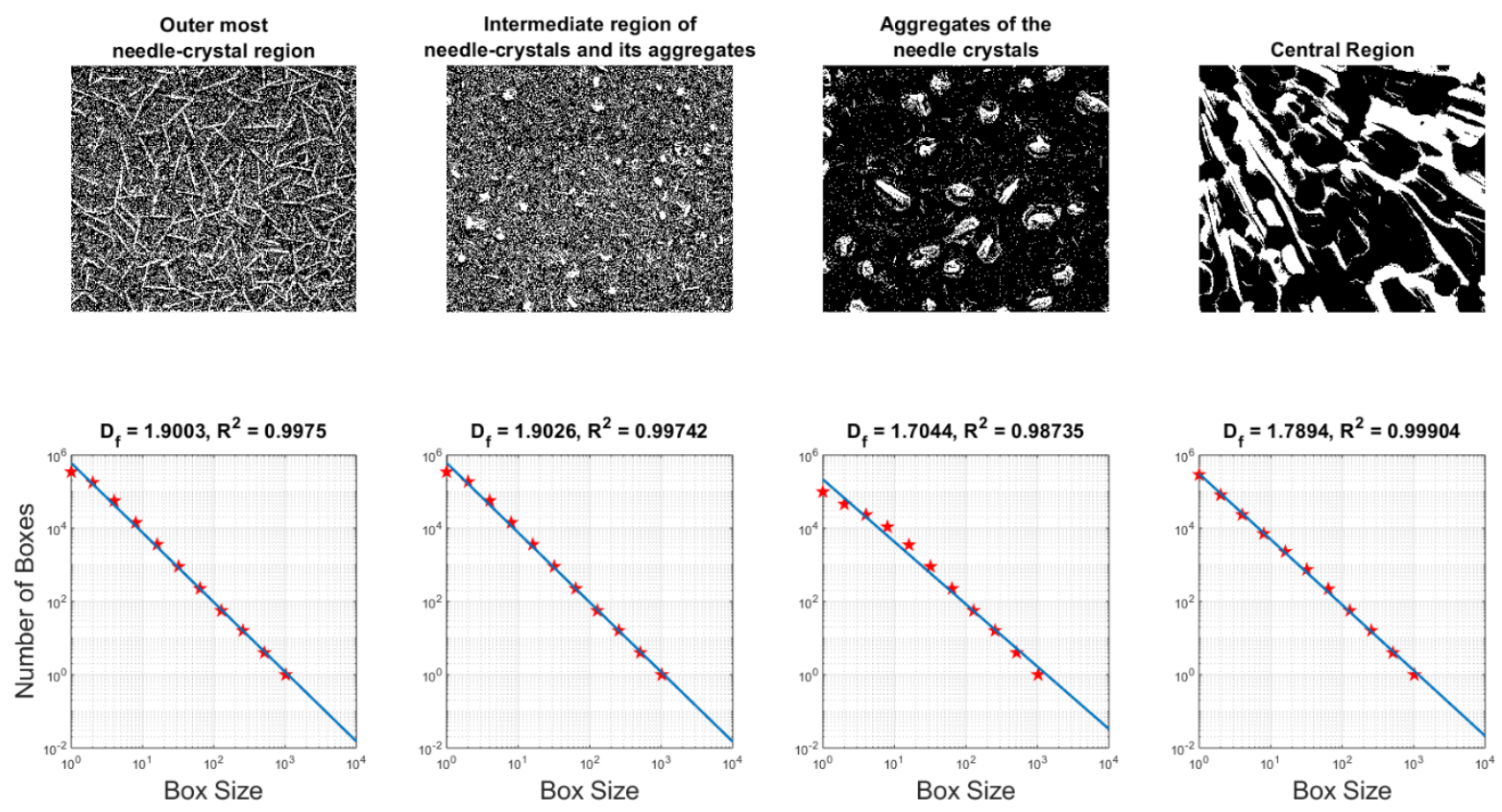

(b) 

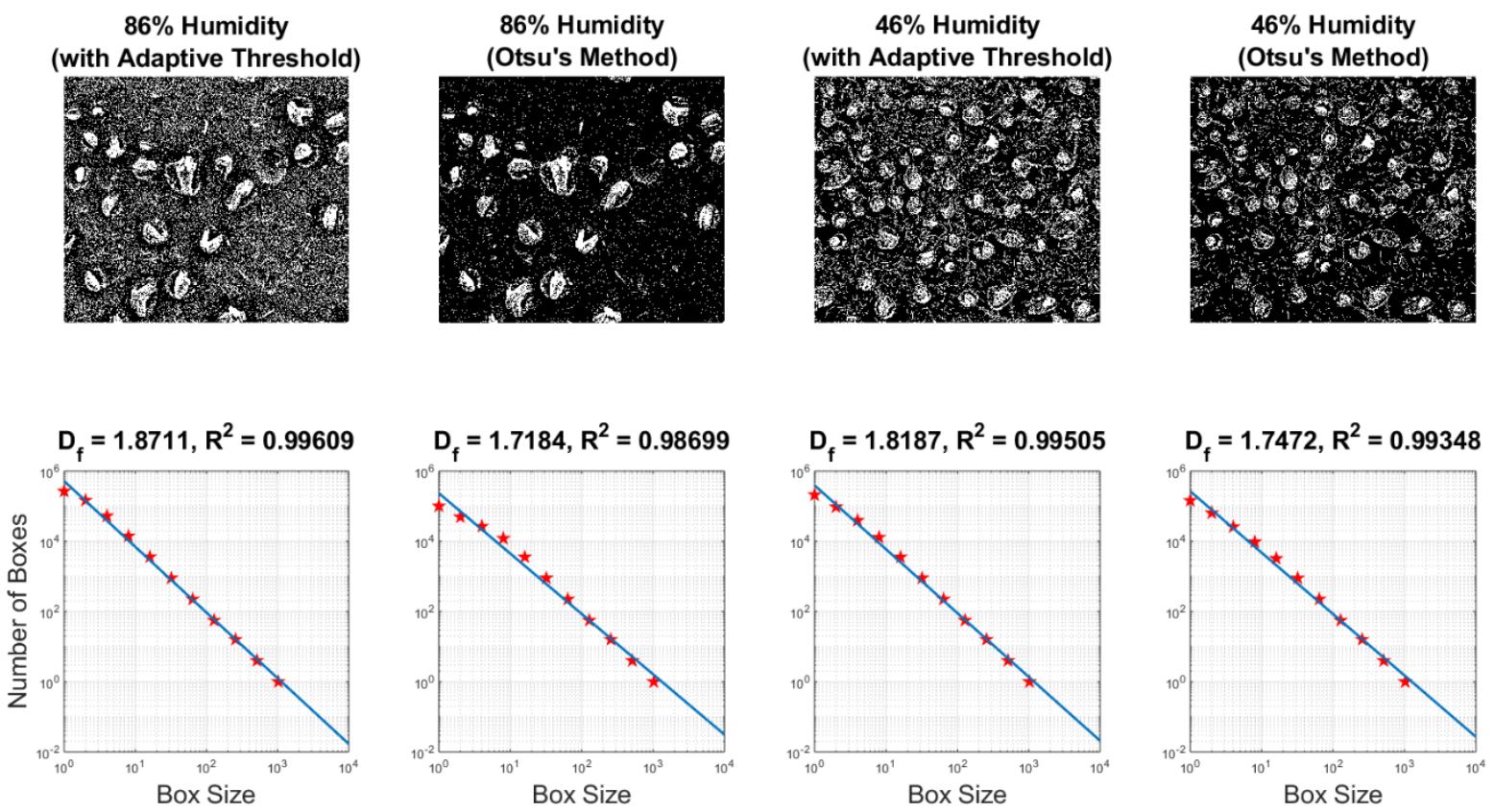

(c)
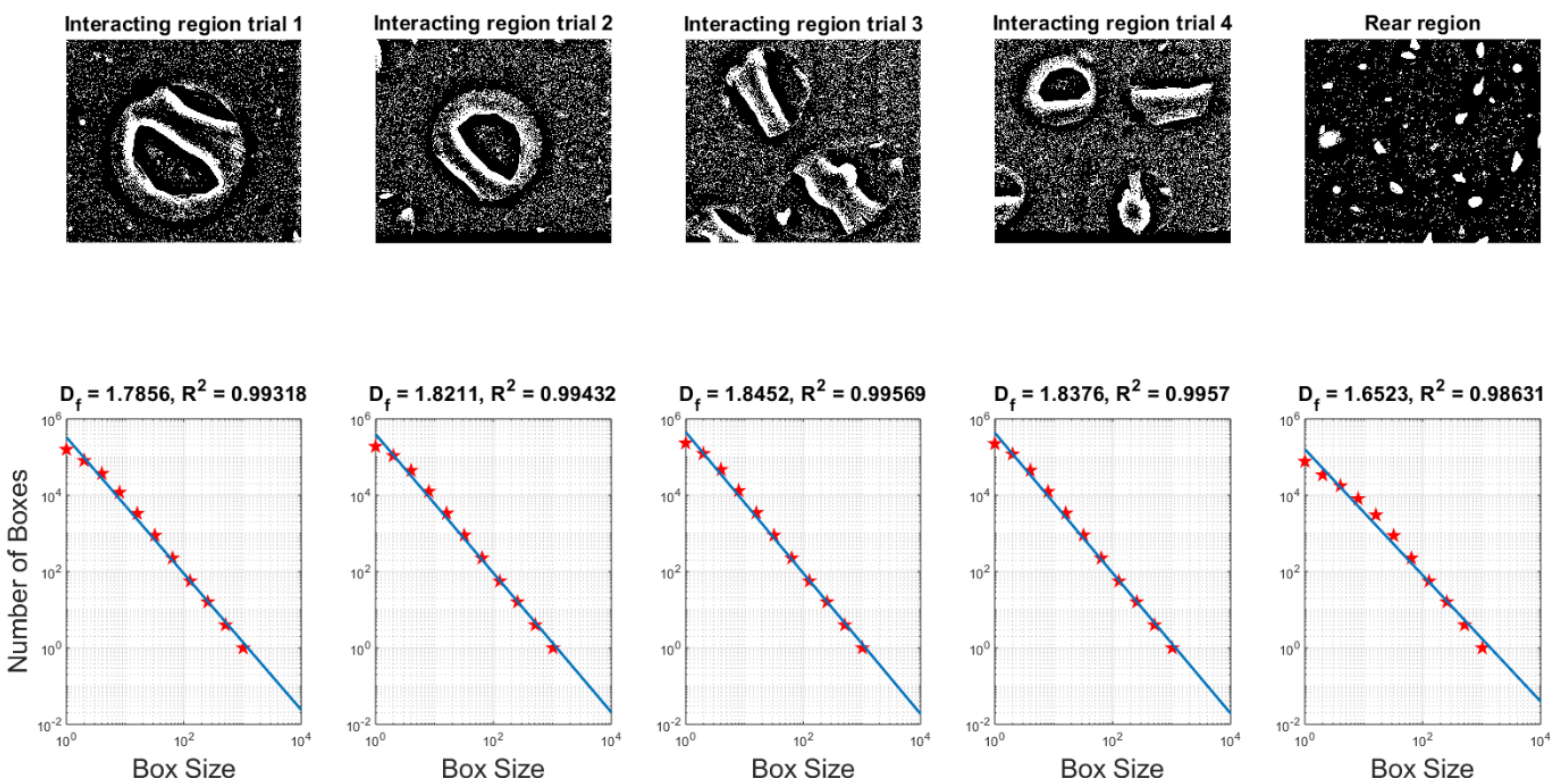

(d) 

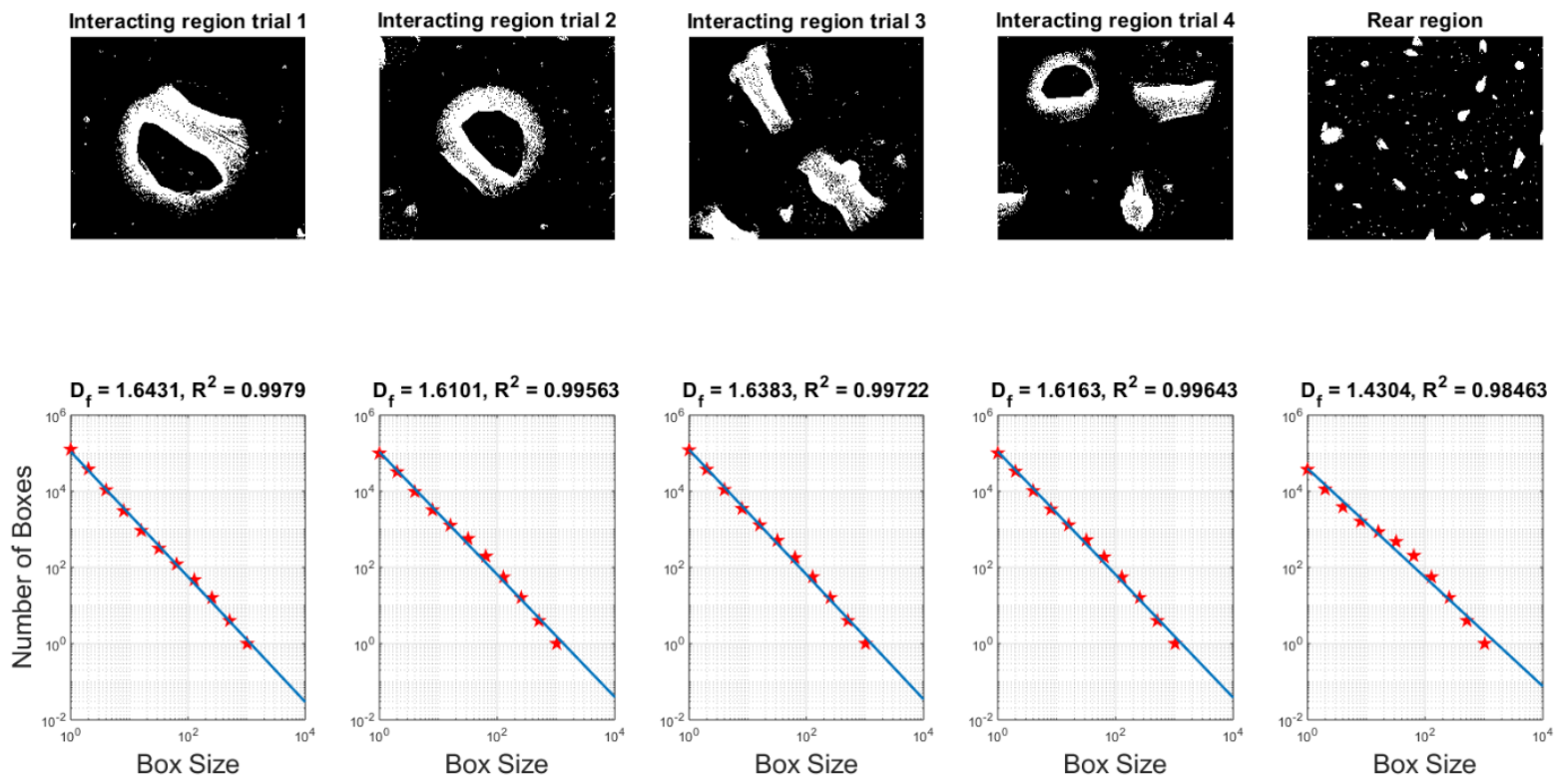

(e)

Figure 11: Fractal analysis (a) single drop with adaptive threshold, (b) single drop with Otsu's method, (c) single drop with humidity effects for Figure 6 with both the method, (d) interacting drop with adaptive threshold, (e) interacting drop with Otsu's method. These figures are the binary versions of the SEM images using two different thresholding methods - Otsu and adaptive thresholding. The corresponding fractal dimension estimation results are depicted in the bottom subpanels along with the goodness of fit $\left(R^{2}\right)$ between box counting vs. box size. Depending on the choice of thresholding method, the background of the blisters are more noisy which might affect the fractal dimension calculation.

\subsection{Characterization of Objects and Their Shapes}

It is interesting to note that each FESEM image has got needle like and other objects of various shapes. One may be interested in finding out the shape statistics and the number distribution of these objects for different regions of the drop and varying with different experimental conditions. For the purpose of object detection and characterisation, we have used the adaptive thresholding method (Bradley \& Roth 2007), to binarize the grayscale image first, followed by identifying moderately large objects in the scene that are connected to 8 neighbouring pixels. We found that adaptive thresholding method preserves the fine structures of the FESEM images compared to the Otsu's method (Otsu 1979). Then using the binary images, several statistical parameters are extracted. Different regions or objects are attributed with different shape parameters e.g. area, perimeter, eccentricity etc.

The eccentricity Ecc $=\sqrt{1-\left(b^{2} / a^{2}\right)}$, is a measure of circularity while $b$ and $a$ being the major and minor axes of the detected objects. The distribution of area, perimeter, eccentricity and areaperimeter relation are compared in Figure 12 for three drop drying conditions using the two thresholding methods - Otsu's and adaptive method both of which show a similar nature of the distributions. It is observed from the distribution of eccentricity for most of the images that there are more objects with $\mathrm{Ecc} \approx 1$ rather than Ecc $\approx 0$, indicating the presence of many long circular objects compared to the number of circular objects.

The shape parameter $(S)$ involving the area vs. perimeter relation is an important parameter indicating the shape of the detected objects. For a circular object $S$ can be represented as: 


$$
S=\frac{4 \pi A}{P^{2}}=\frac{4 \pi \cdot \pi r^{2}}{(2 \pi r)^{2}}=\frac{4 \pi^{2} r^{2}}{4 \pi^{2} r^{2}}=1
$$

with $r$ being the radius of the object.

Previous studies on fractal objects like cloud and rivers in (Feder 2013) have shown $P \gg A$ for fractal structures. Therefore, for fractal objects in the FESEM images we expect to get $S=\frac{4 \pi A}{P^{2}} \rightarrow 0$, as evident from the distributions of area/perimeter relation in Figure 12(a)-(b). The eccentricity together with area/perimeter relation can be useful in discriminating compactness or roundedness vs. fractal nature of the objects.
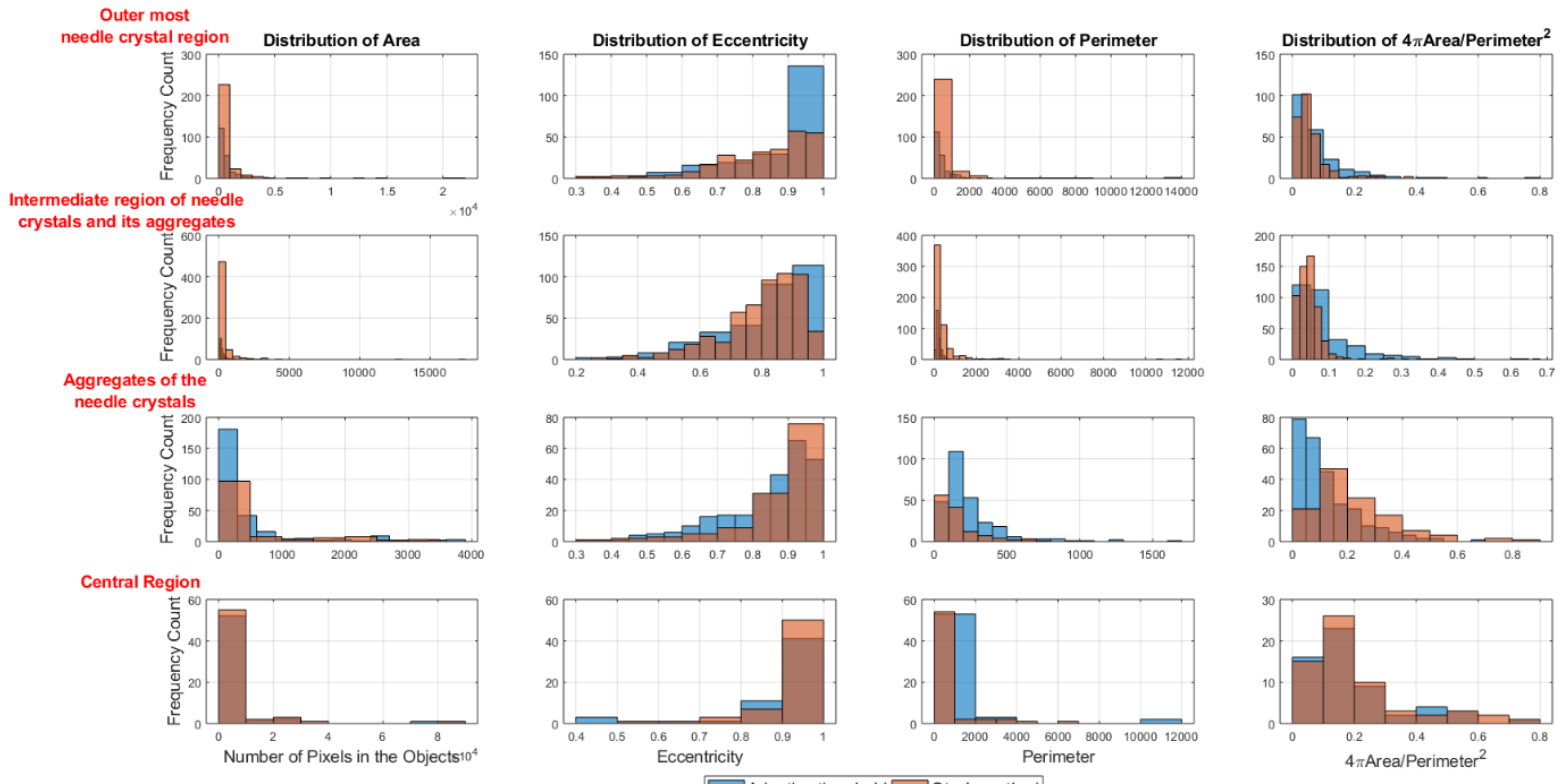

(a)
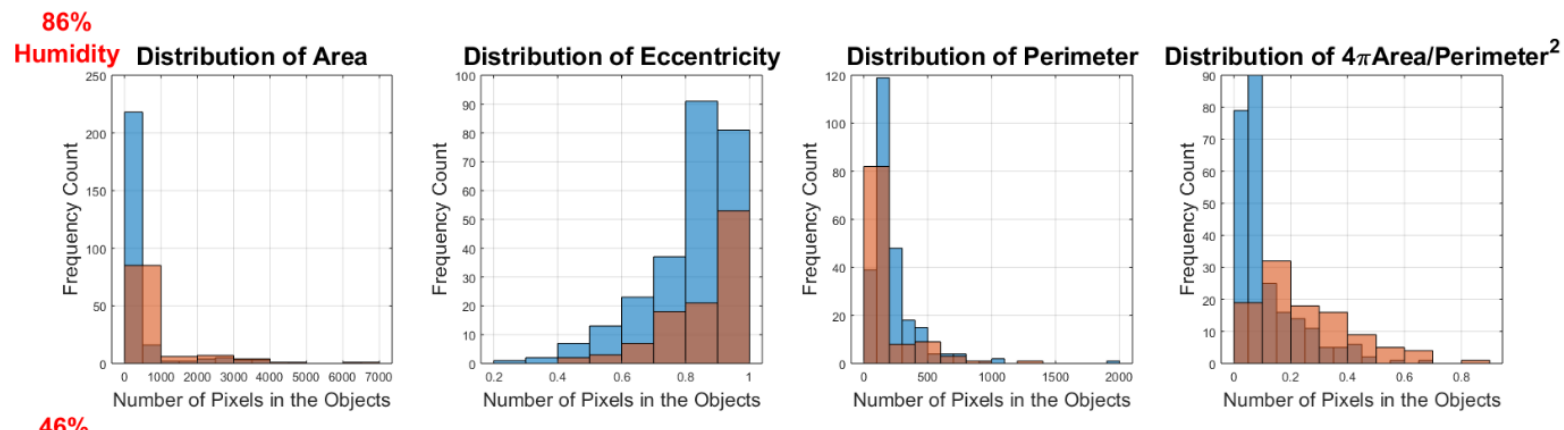

Humidity Distribution of Area
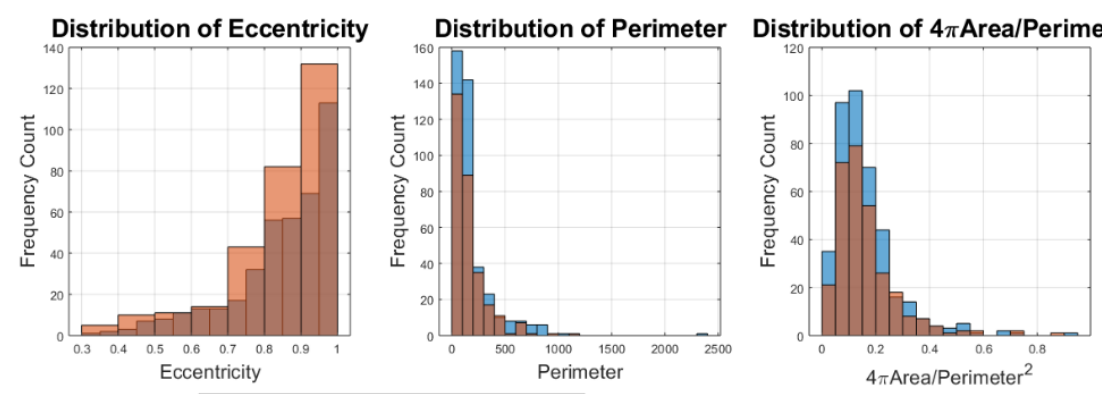
(b)
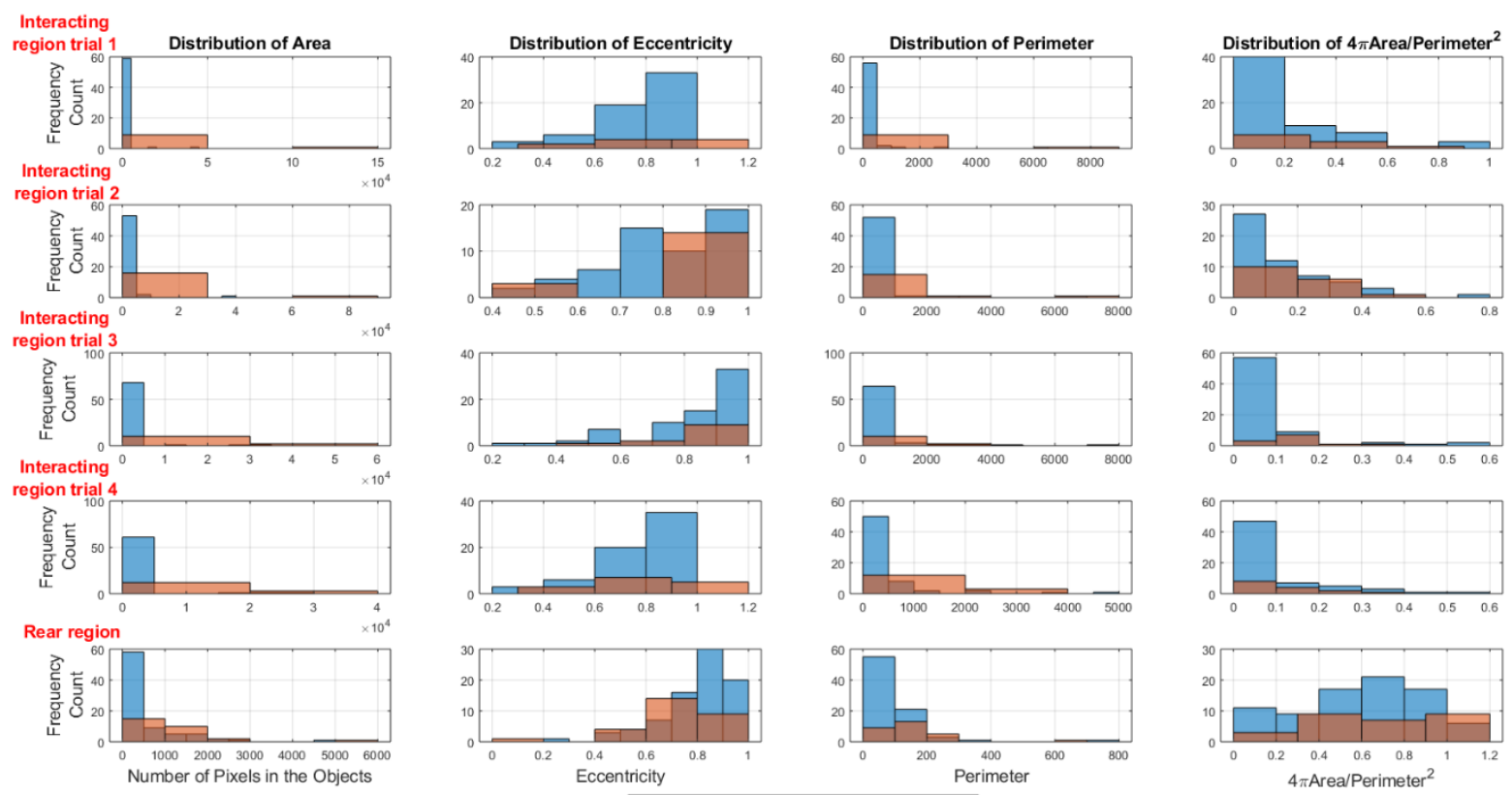

$\square$ Adaptive threshold $\square$ Otsu's method

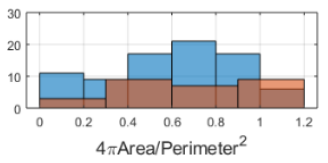

(c)

Figure 12: Distribution of objects for (a) single drop analysis, (b) single drop with change in humidity, (c) interacting drop.

\subsection{Feature Correlation Analysis and Resampling}

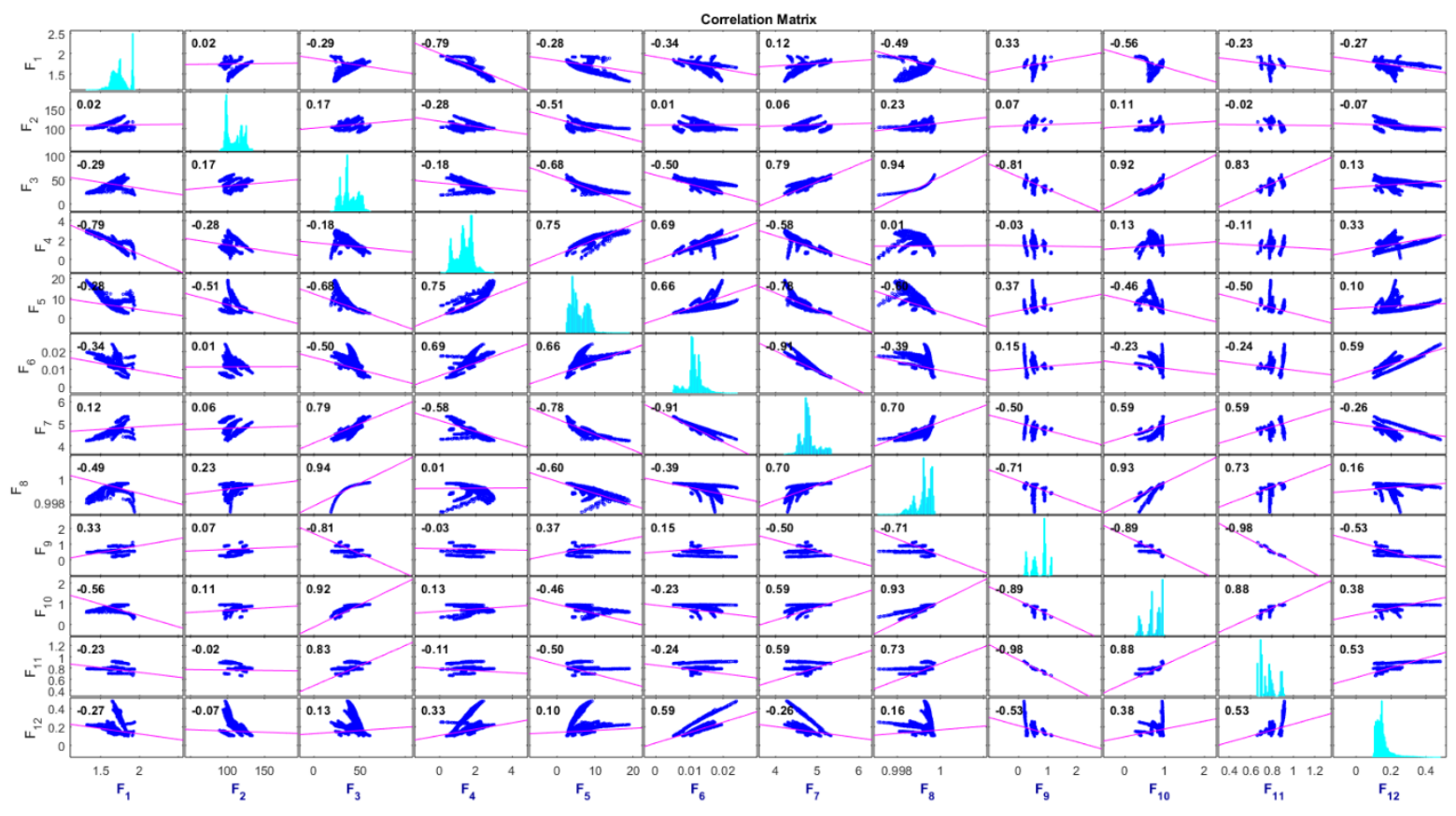

Figure 13: Correlation between the feature pairs for all the FESEM images.

Next we calculate any potential redundant information in the calculated features in all the datasets of FESEM images using the sliding window feature extraction (Dutta Sinha et al. 2017)(Chatterjee et al. 2017). The calculated features are $F_{1}=$ fractal dimension $\left(D_{f}\right), F_{2}=$ pixel intensity mean $(\mu), F_{3}=$ standard deviation $(\sigma), F_{4}=$ skewness $(\gamma), F_{5}=$ kurtosis $(\beta), F_{6}=\operatorname{energy}(E), F_{7}=$ entropy $(H), F_{8}=$ 
smoothness, $F_{9}=$ contrast, $F_{10}=$ spatial correlation, $F_{11}=$ homogeneity, $F_{12}=$ uniformity or $2 \mathrm{D}$ energy. Due to the lack of many independent experiments on pattern formation and FESEM imaging, previous studies like (Dutta Sinha et al. 2017)(Chatterjee et al. 2017) have used resampling approaches on large images or long signals with smaller windows to calculate features from different parts of the same data and consider them to be independent experimental datasets for statistical comparison. The above features from each FESEM images were resampled using a sliding window in $x$ and $y$-direction by 10 pixels to calculate the sliding window statistics. The maximum size of the window is chosen as $2^{N}$ with $N=9$, since a larger power exceeds the length of the image in either $x$ or $y$-direction. These are helpful in understanding potential correlation between the features in different datasets (when combined together) and also allows one to test several hypotheses based on the calculated features. The feature correlations between the FESEM images under consideration are shown in Figure 13 which shows there are significantly high correlation $(|r|>0.9)$ between pairs $F_{9}$ (contrast) and $F_{11}$ (2D energy). Rest of the feature pairs show different complicated nonlinear correlation structures.

\subsection{Class Separability Analysis and Hypothesis Testing}

Next, we test the following three hypotheses to find out the significant features indicating the difference between the group of drop drying images:

- Comparison of the 4 regions (outermost, intermediate, aggregates, central) of single drop using analysis of variance (ANOVA) and its non-parametric version

- Comparison of the 2 humidity effects $(86 \%$ and $46 \%$ ) on single drop drying using $t$-test and its non-parametric version

- Comparison of the 2 regions (rear and interacting) of the interacting drop using $t$-test and its non-parametric version

In all the above tests, the null hypothesis is considered to be that there is no significant difference in the groups against the alternative hypothesis that there is significant difference and for all the cases, the significance level is set to 0.05 as default. Both parametric and nonparametric hypothesis tests are conducted for comparing three regions of the single drop's 3 regions viz. analysis of variance (ANOVA) and Kruskal-Wallis test. This is due to the fact that not all of the distributions of features in the boxplots in Figure 14(b) do not look close to the normal distribution and the joint and marginal distributions are found to be multimodal in nature for Figure 15(a). For comparing two groups for the humidity effects $(86 \%$ and $46 \%)$ and interacting vs. rear region of the interacting drops, we use the $t$ test and its non-parametric version Wilcoxon rank sum test which is also equivalent to the MannWhitney U-test (Gibbons \& Chakraborti 2010). More details of these parametric and non-parametric tests can be found in (Field et al. 2012). As similar comparison has also been done using the for the interacting drop's different regions.

Using these tests, one can easily identify the prominent statistical features that shows significant difference between these groups viz. experimental conditions or different regions of the drops. For comparing more groups using some chosen features, usually the scatter matrix $(J)$ is used:

$$
J=\operatorname{trace}\left\{S_{w}^{-1} S_{b}\right\} .
$$

Here, $\left\{S_{w}, S_{b}\right\}$ indicate the within class and between class scatter matrix and can be calculated as: 


$$
\begin{aligned}
& S_{w}=\sum_{i=1}^{M} P_{i} S_{i}, \\
& S_{b}=\sum_{i=1}^{M} P_{i}\left(m_{i}-m_{0}\right)\left(m_{i}-m_{0}\right)^{T} .
\end{aligned}
$$

In (6), $m_{0}$ is the global mean over all classes, $M$ is the number of class, $\left\{m_{i}, S_{i}\right\}$ are the mean and covariance of class $i$. Also, $P_{i}$ is the a-priori probability of each class $C_{i}$ i.e. $P_{i}=1 / M$ for balanced data and $P_{i} \simeq n_{i} / N$ for unbalanced data with $\left\{n_{i}, N\right\}$ being the number of data-points in a particular class and total number of data-points respectively. It has been shown in (Theodoridis et al. 2010) that for a two class problem the scatter matrix $(J)$ can be interpreted as the Fisher's discriminant ration (FDR):

$$
\mathrm{FDR}=\frac{\left(m_{1}-m_{2}\right)^{2}}{\sigma_{1}^{2}+\sigma_{2}^{2}},
$$

where the covariances become variances of the two classes.

Table 1: Non-parametric and parametric hypothesis tests for single drop comparison

\begin{tabular}{|c|c|c|c|c|c|}
\hline & & \multicolumn{2}{|c|}{ Kruskal-Wallis test } & \multicolumn{2}{c|}{ ANOVA } \\
\cline { 3 - 6 } Feature & Scatter Matrix & $\chi^{2}$ statistic & $\boldsymbol{p}$-value & $\boldsymbol{F}$ statistic & $\boldsymbol{p}$-value \\
\hline$F_{11}=$ homogeneity & 1058.16 & 7214.06 & 0 & 2765217.33 & 0 \\
\hline$F_{9}=$ contrast & 695.61 & 7078.84 & 0 & 1804488.49 & 0 \\
\hline$F_{10}=$ spatial correlation & 76.40 & 6492.73 & 0 & 195804.68 & 0 \\
\hline$F_{3}=$ standard deviation $(\sigma)$ & 47.23 & 7211.70 & 0 & 123060.14 & 0 \\
\hline$F_{7}=$ entropy $(H)$ & 29.47 & 6788.66 & 0 & 77609.69 & 0 \\
\hline$F_{8}=$ smoothness & 28.10 & 7211.70 & 0 & 72383.03 & 0 \\
\hline$F_{6}=$ energy $(E)$ & 25.56 & 7112.14 & 0 & 67786.08 & 0 \\
\hline$F_{5}=$ kurtosis $(\beta)$ & 22.90 & 6704.09 & 0 & 58984.49 & 0 \\
\hline$F_{2}=$ mean $(\mu)$ & 16.18 & 4552.80 & 0 & 41523.03 & 0 \\
\hline$F_{1}=$ fractal dimension $\left(D_{f}\right)$ & 12.99 & 6388.12 & 0 & 33640.26 & 0 \\
\hline$F_{4}=$ skewness $(\gamma)$ & 6.55 & 6429.71 & 0 & 16972.35 & 0 \\
\hline$F_{12}=$ uniformity or 2D energy & 1.98 & 5003.71 & 0 & 5168.67 & 0 \\
\hline
\end{tabular}

Using the scatter matrix the features are first ranked before they were used for the hypothesis testing which are shown in Table 1-Table 3 for the three different comparisons, discussed above. The most significant features for each hypothesis are also listed along with their $p$-values which shows the null hypothesis gets rejected and alternative hypothesis is favoured. To visualize these difference using the most significant features after ranked using scatter matrix and rejecting the null hypothesis, the corresponding joint distributions using the most significant feature pairs in each cases are shown in Figure 14(a), Figure 15(a) and Figure 16(a) respectively, along with the marginal distributions. However, a through comparison on individual features are shown in the respective boxplots in Figure 14(b), Figure 15(b) and Figure 16(b). 
Table 2: Non-parametric and parametric hypothesis tests for single drop with humidity effect comparison

\begin{tabular}{|c|c|c|c|c|c|}
\hline & & \multicolumn{2}{|c|}{ Wilcoxon rank sum test } & \multicolumn{2}{c|}{ Two sample $\boldsymbol{t}$-test } \\
\cline { 3 - 6 } Feature & Scatter Matrix & $\boldsymbol{z}$ statistic & $\boldsymbol{p}$-value & $\boldsymbol{t}$ statistic & $\boldsymbol{p}$-value \\
\hline$F_{2}=$ mean $(\mu)$ & 57.35 & -54.44 & 0 & -476.09 & 0 \\
\hline$F_{11}$ homogeneity & 18.36 & -54.44 & 0 & -269.38 & 0 \\
\hline$F_{4}$ skewness $(\gamma)$ & 10.42 & 54.44 & 0 & 202.92 & 0 \\
\hline$F_{5}$ kurtosis $(\beta)$ & 6.84 & 54.44 & 0 & 164.42 & 0 \\
\hline$F_{12}$ uniformity or 2 D energy & 2.78 & -54.22 & 0 & -104.85 & 0 \\
\hline$F_{1}$ fractal dimension $\left(D_{f}\right)$ & 0.70 & -44.32 & 0 & -52.57 & 0 \\
\hline$F_{10}$ spatial correlation & 0.53 & -40.00 & 0 & -45.87 & 0 \\
\hline$F_{7}$ entropy $(H)$ & 0.46 & -34.27 & $2.56 \times 10^{-257}$ & -42.46 & 0 \\
\hline$F_{3}$ standard deviation $(\sigma)$ & 0.35 & -30.73 & $2.34 \times 10^{-207}$ & -37.40 & $2.50 \times 10^{-262}$ \\
\hline$F_{8}$ smoothness & 0.34 & -30.73 & $2.34 \times 10^{-207}$ & -36.59 & $1.05 \times 10^{-252}$ \\
\hline$F_{9}$ contrast & 0.04 & 9.12 & $7.42 \times 10^{-20}$ & 12.33 & $2.52 \times 10^{-34}$ \\
\hline$F_{6}$ energy $(E)$ & 0.01 & 4.49 & $7.14 \times 10^{-06}$ & 4.89 & $1.05 \times 10^{-06}$ \\
\hline
\end{tabular}

Table 3: Non-parametric and parametric hypothesis tests for interacting drop comparison

\begin{tabular}{|c|c|c|c|c|c|}
\hline & & \multicolumn{2}{|c|}{ Wilcoxon rank sum test } & \multicolumn{2}{c|}{ Two sample t-test } \\
\cline { 3 - 6 } Feature & Scatter Matrix & $\boldsymbol{z}$ statistic & $\boldsymbol{p}$-value & $\boldsymbol{t}$ statistic & $\boldsymbol{p}$-value \\
\hline$F_{12}$ uniformity or $2 \mathrm{D}$ & & & & & \\
energy & 38.42 & -68.86 & 0 & -410.03 & 0 \\
\hline$F_{6}$ energy $(E)$ & 27.74 & -68.86 & 0 & -375.37 & 0 \\
\hline$F_{7}$ entropy $(H)$ & 15.71 & 68.86 & 0 & 258.38 & 0 \\
\hline$F_{4}$ skewness $(\gamma)$ & 10.38 & -68.86 & 0 & -246.91 & 0 \\
\hline$F_{5}$ kurtosis $(\beta)$ & 8.96 & -68.85 & 0 & -294.26 & 0 \\
\hline$F_{3}$ standard deviation $(\sigma)$ & 7.05 & 68.56 & 0 & 174.35 & 0 \\
\hline$F_{1}$ fractal dimension $\left(D_{f}\right)$ & 5.56 & 68.44 & 0 & 156.56 & 0 \\
\hline$F_{8}$ smoothness & 4.37 & 68.56 & 0 & 226.82 & 0 \\
\hline$F_{11}$ homogeneity & 4.23 & -68.86 & 0 & -129.44 & 0 \\
\hline$F_{9}$ contrast & 3.73 & 68.86 & 0 & 121.59 & 0 \\
\hline$F_{2}$ mean $(\mu)$ & 0.09 & 20.73 & $2.01 \times 10^{-95}$ & 18.66 & $2.09 \times 10^{-76}$ \\
\hline$F_{10}$ spatial correlation & 0.03 & 18.43 & $8.01 \times 10^{-76}$ & 12.30 & $1.55 \times 10^{-34}$ \\
\hline
\end{tabular}




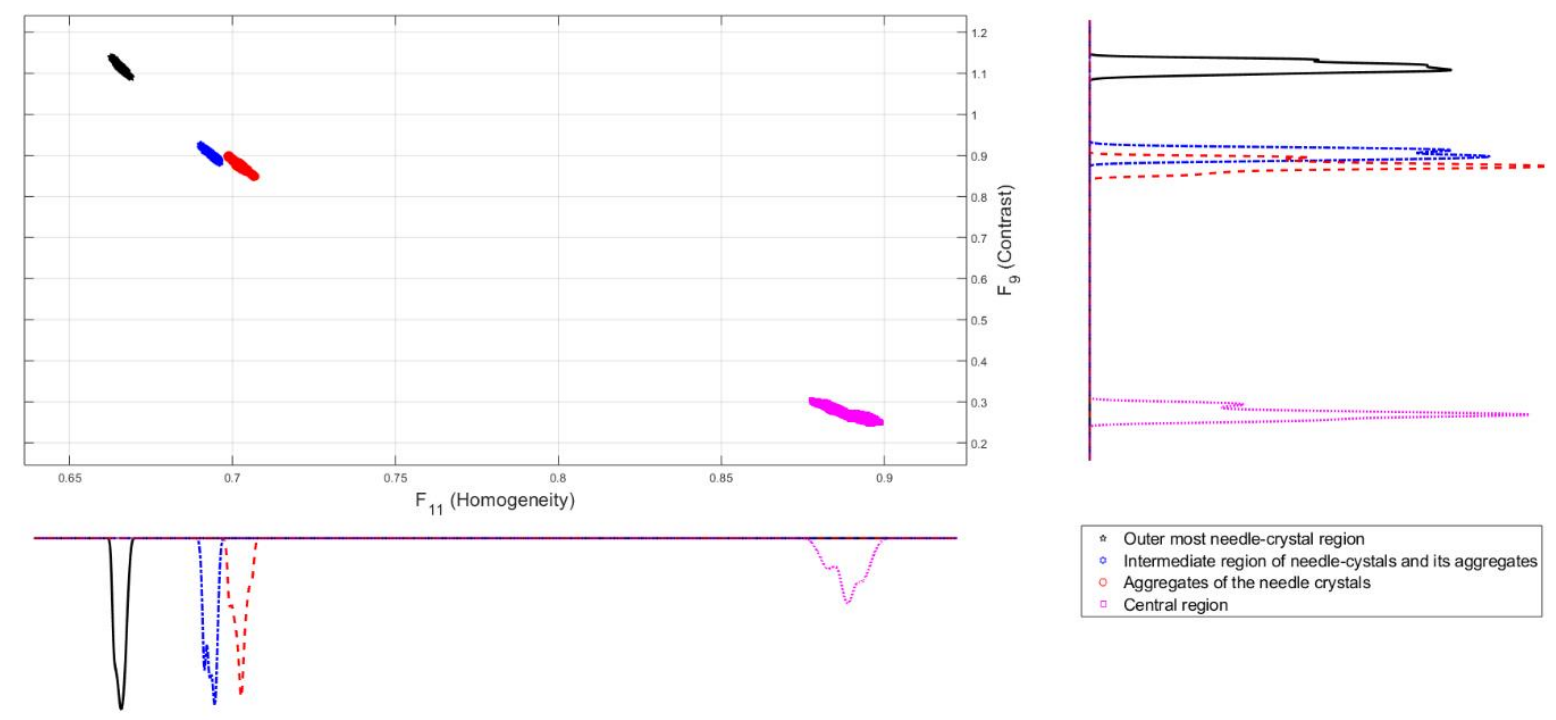

(a)
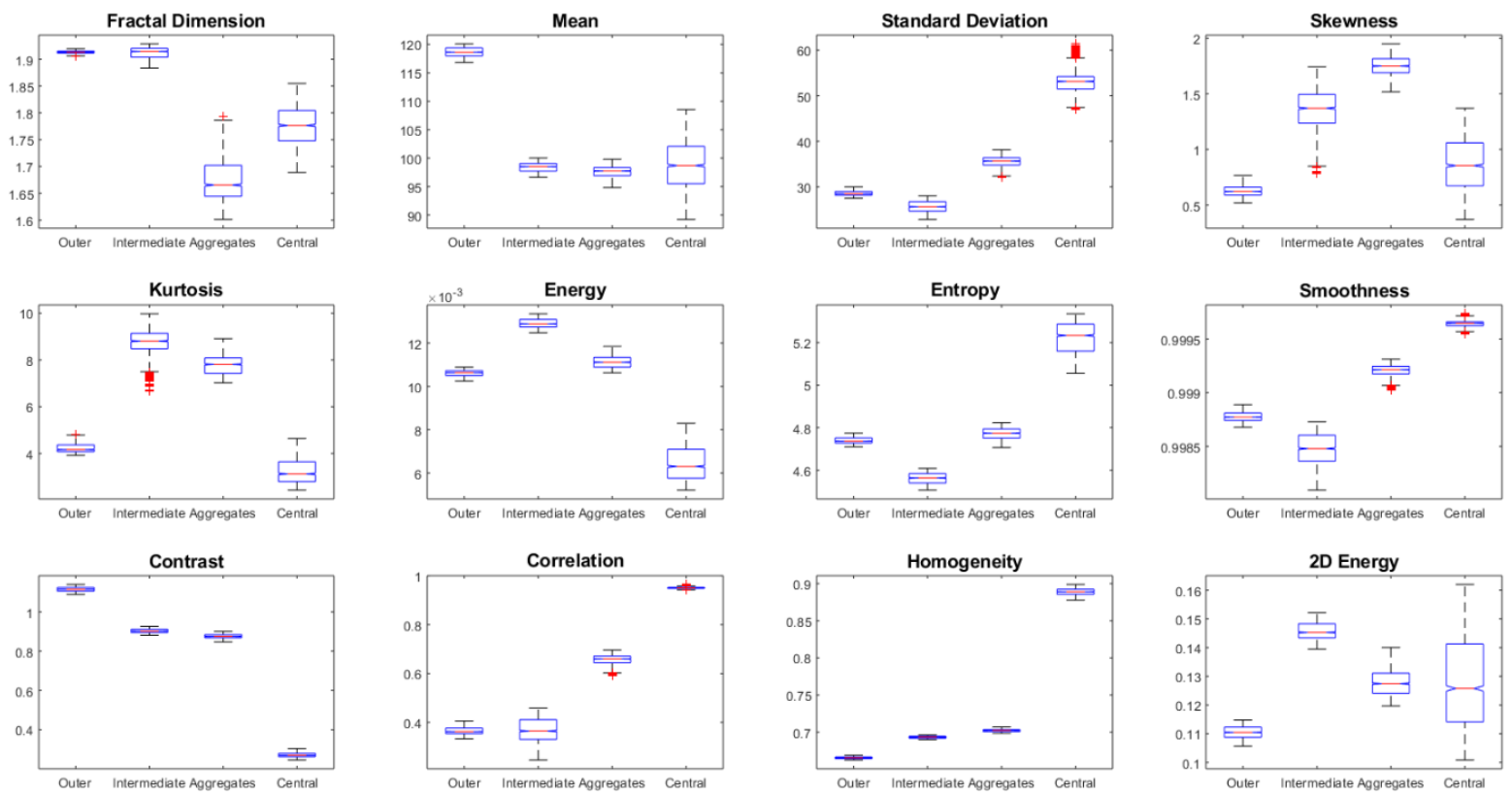

(b)

Figure 14: Comparison of the different parts of the single drop (a) using the top two ranked features, (b) using all the 12 features. 


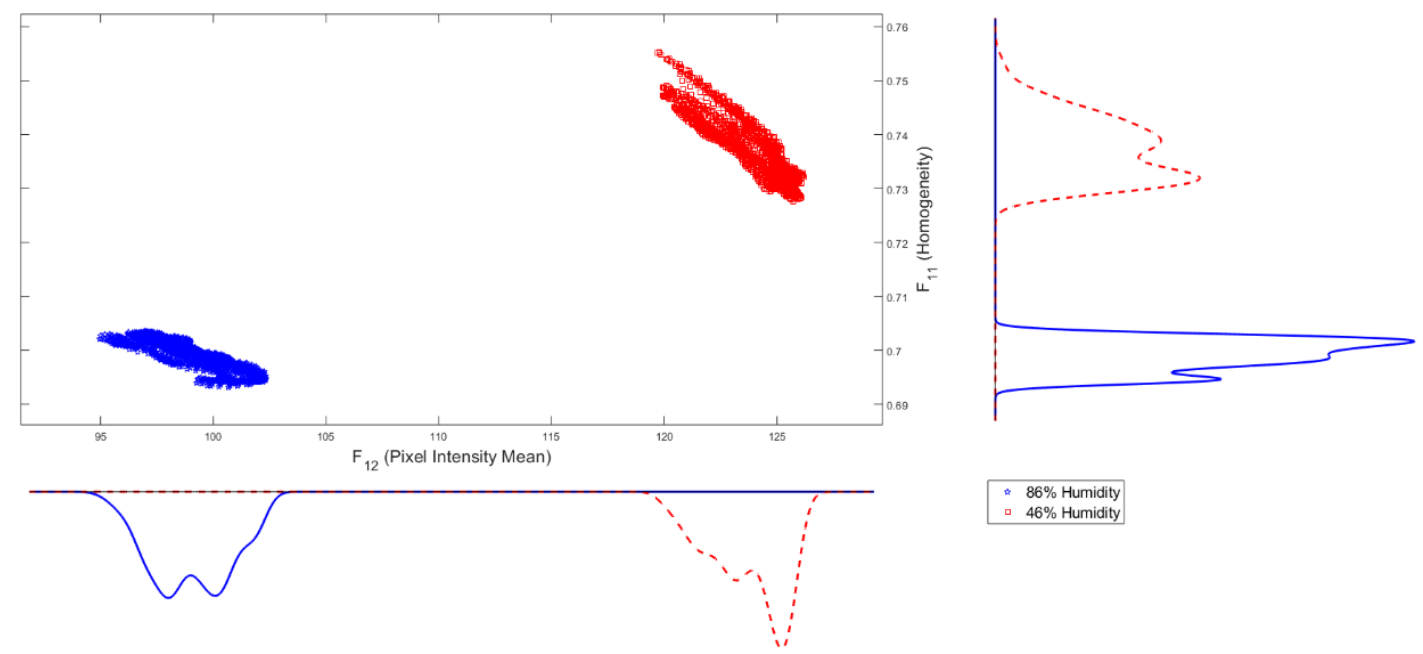

(a)
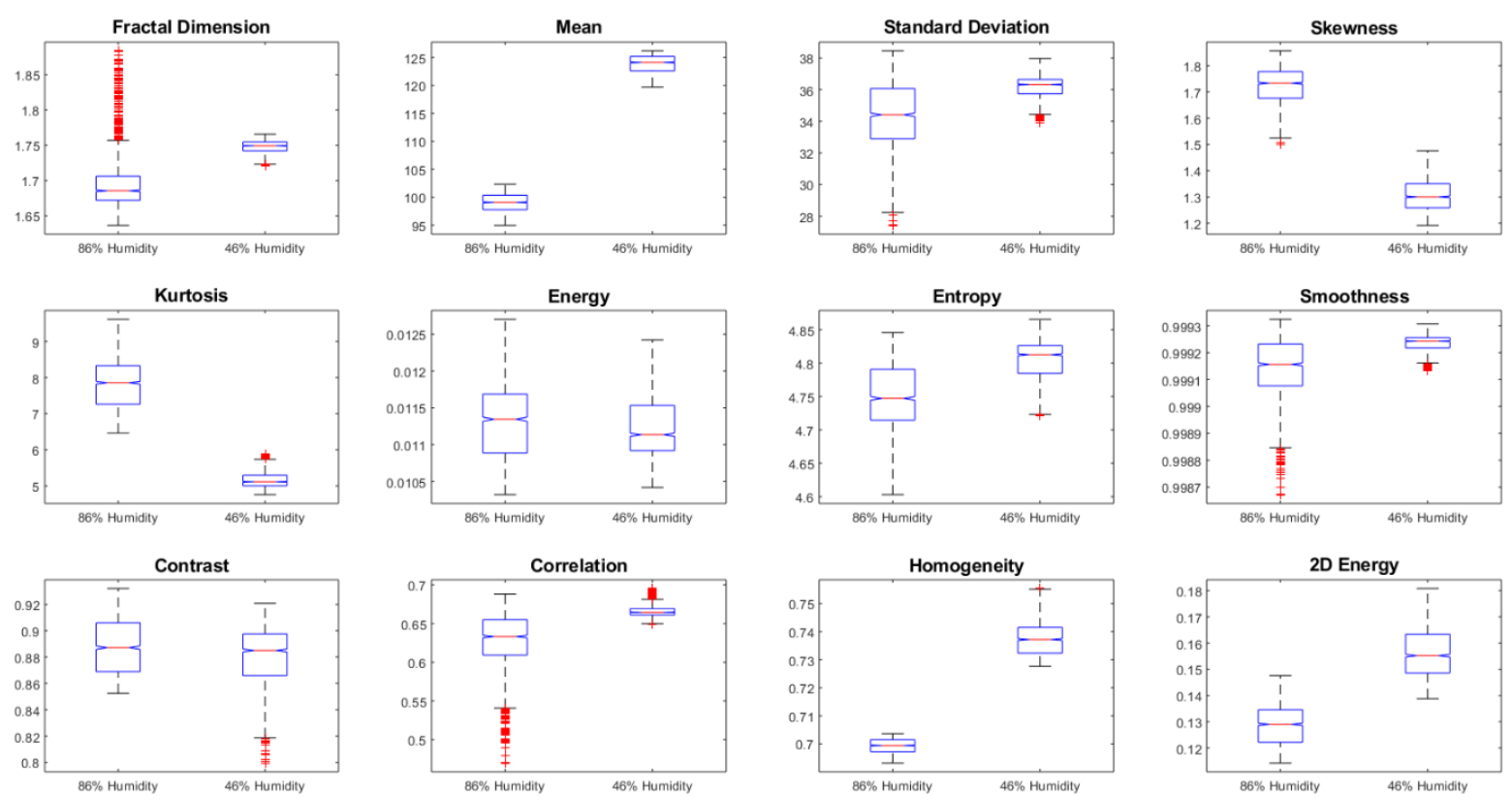

(b)

Figure 15: Comparison of the different parts of the single drop with humidity effects (a) using the top two ranked features, (b) using all the 12 features. 


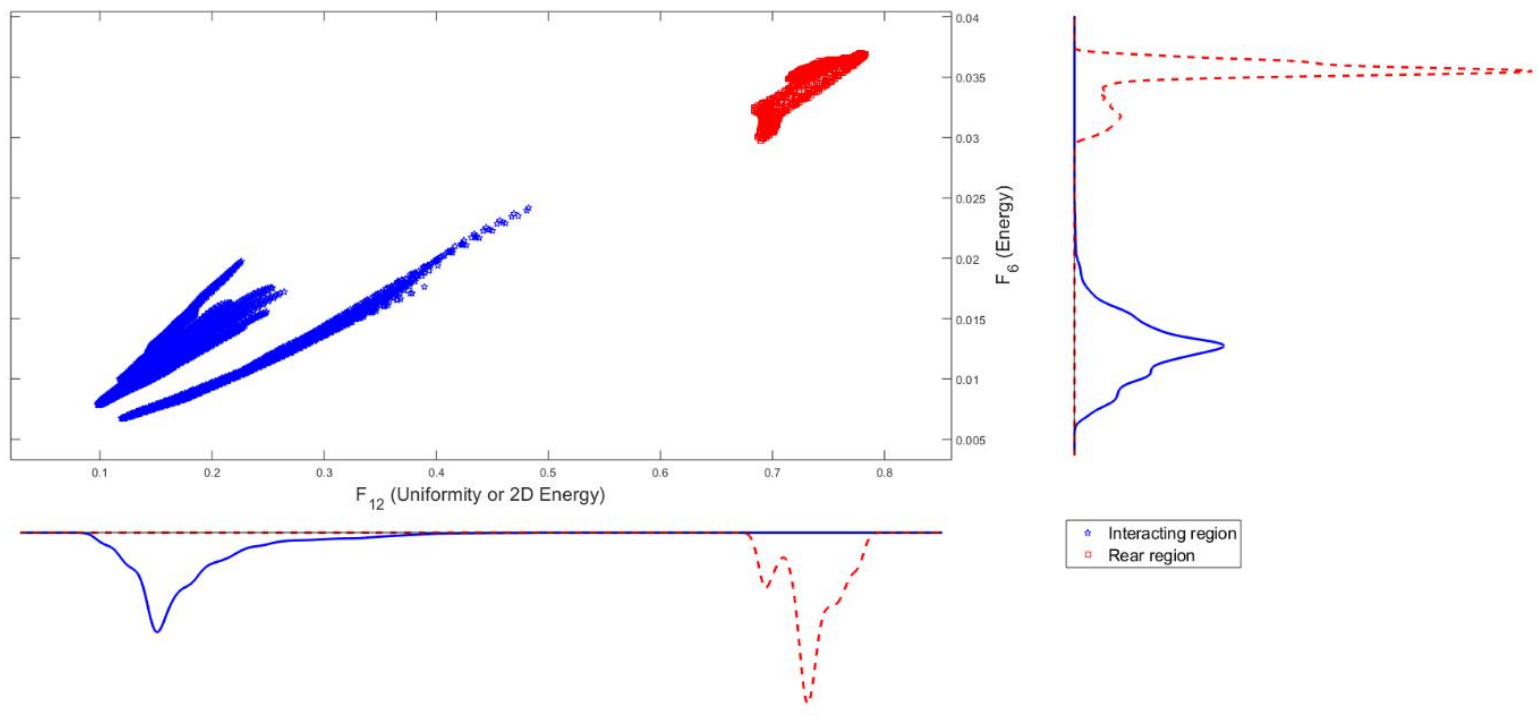

(a)
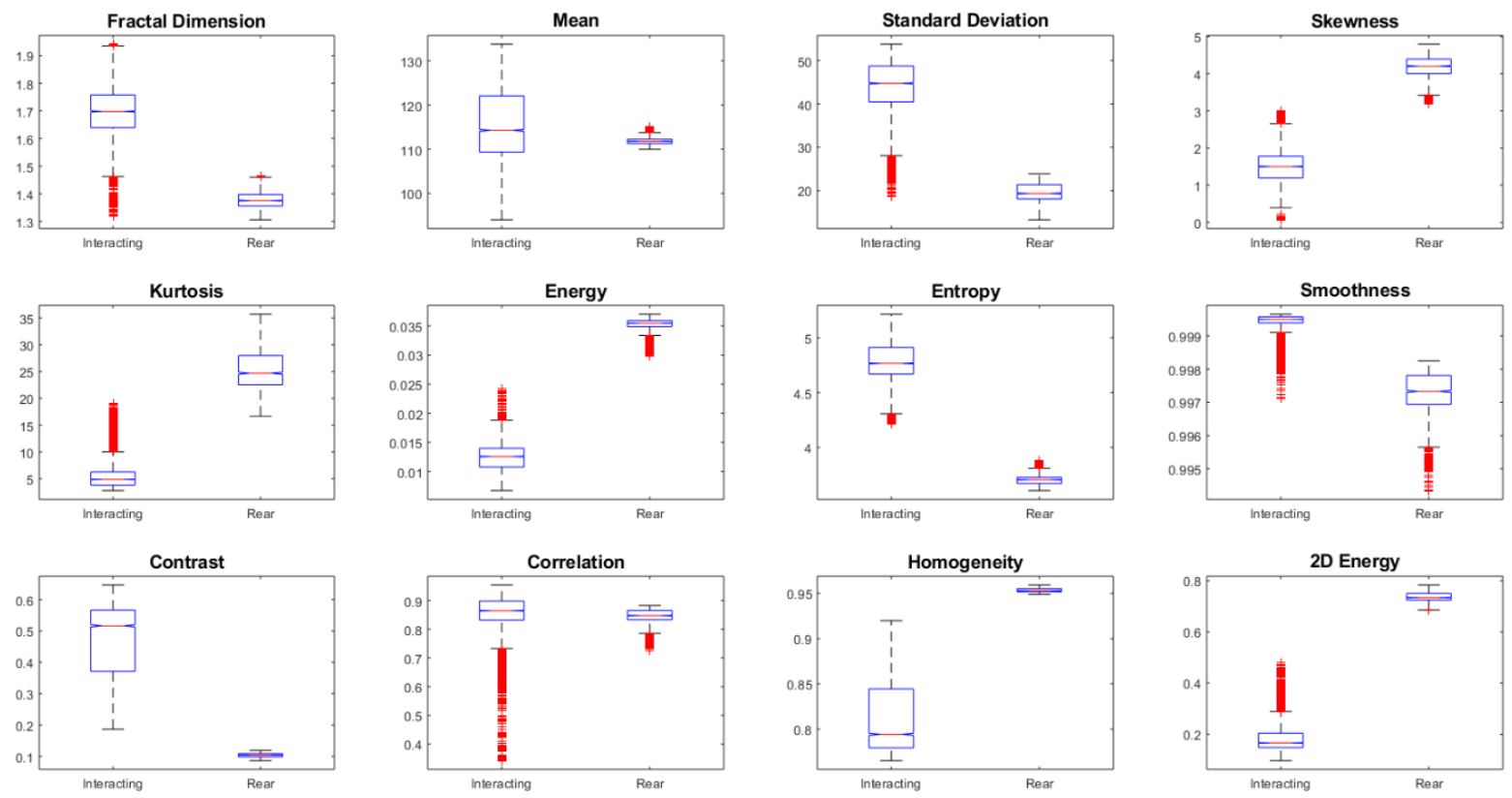

(b)

Figure 16: Comparison of the different parts of the interacting drops (a) using the top two ranked features, (b) using all the 12 features.

\section{Conclusions}

Here we show the pattern formation in crystals for drop drying with salts in presence of gelatin. We have studied the pattern formation of single isolated drying drop as well as two interacting drops of gelatin solution containing $\mathrm{Na}_{2} \mathrm{SO}_{4}$. We have shown that the deposition pattern of dried up drops of $\mathrm{Na}_{2} \mathrm{SO}_{4}$ differs significantly when gelatin is added to the solution. Many distinct concentric regions of different widths have been found. A possible mechanism of the formation of patterns in the drying drops is also proposed. Here we present a detailed study on the effect of a neighbouring drop on the deposition pattern, morphology and crystal growth of the adjacent drop. We find pronounced effect in the nature of the crystal growth of sodium sulfate in dried interacting drops when the drop separation 
is less than $350 \mu \mathrm{m}$. The needles aggregate and form lumps or clusters in the close proximity region of the drops. But in the rear region, we have observed small needle crystals of sodium sulfate. These results are consistent and in agreement with our proposed mechanism. Non-uniformity in width of the outer most layer in drying interacting drops are found due to the non-uniform rate of evaporation from the drop surface. This non-uniformity observed in interacting drops fade away as the separating distance is increased. Hence, separating distance between drops controls the local relative humidity surrounding the drop. This affects the pattern formation inside the interacting drops.

Thus, the regulation of the local ambient relative humidity of a drop in the presence of another drop might have implications in particle separation, protein separation and size distribution of the saltprotein aggregates. The interacting drop configuration might also be a useful tool in the field of pathological and forensic diagnosis. An interesting outlook of this work would be to analyse theoretical studies of crystal growths of the salt in these interacting drops.

The paper also identifies the most significant statistical features out of the textural (first and second order statistics) and fractal analysis. We also show the difference in the shape distributions for different objects and the number distribution of the objects in different regions of the drops and also their variation with humidity. In future, mathematical modelling of drop drying can be investigated and also using a larger volume of datasets with repeated experiments under similar conditions.

\section{Acknowledgments}

MDC thanks SERB, India for providing national post-doctoral fellowship (PDF/2016/001151/PMS). BR is grateful to DST, Govt. of India for funding this research through project No. SR/S2/CMP127/2012. BR thanks DST for providing a Junior Research fellowship.

\section{References}

Alvarez-Puebla, R.A. et al., 2011. Gold nanorods 3D-supercrystals as surface enhanced Raman scattering spectroscopy substrates for the rapid detection of scrambled prions. Proceedings of the National Academy of Sciences, 108(20), pp.8157-8161.

Arifin, A.Z. \& Asano, A., 2006. Image segmentation by histogram thresholding using hierarchical cluster analysis. Pattern Recognition Letters, 27(13), pp.1515-1521.

Bradley, D. \& Roth, G., 2007. Adaptive thresholding using the integral image. Journal of graphics tools, 12(2), pp.13-21.

Brutin, D. et al., 2011. Pattern formation in drying drops of blood. Journal of Fluid Mechanics, 667, pp.85-95.

Chatterjee, S.K. et al., 2017. Comparison of decision tree based classification strategies to detect external chemical stimuli from raw and filtered plant electrical response. Sensors and Actuators B: Chemical, 249, pp.278-295.

Chen, L. \& Evans, J.R., 2009. Arched structures created by colloidal droplets as they dry. Langmuir, 25(19), pp.11299-11301.

Choudhury, M.D., Das, S. \& Tarafdar, S., 2016. Effect of loading history on visco-elastic potato starch gel. Colloids and Surfaces A: Physicochemical and Engineering Aspects, 492, pp.47-53. 
Choudhury, M.D., Dutta, T. \& Tarafdar, S., 2015. Growth kinetics of $\mathrm{NaCl}$ crystals in a drying drop of gelatin: transition from faceted to dendritic growth. Soft Matter, 11(35), pp.6938-6947.

Choudhury, M.D., Dutta, T. \& Tarafdar, S., 2013. Pattern formation in droplets of starch gels containing $\mathrm{NaCl}$ dried on different surfaces. Colloids and Surfaces A: Physicochemical and Engineering Aspects, 432, pp.110-118.

Deegan, R.D. et al., 1997. Capillary flow as the cause of ring stains from dried liquid drops. Nature, 389(6653), pp.827-829.

Dong, A. et al., 2010. Binary nanocrystal superlattice membranes self-assembled at the liquid-air interface. Nature, 466(7305), p.474.

Dutta Sinha, S. et al., 2017. Monitoring of Wild Pseudomonas Biofilm Strain Conditions Using Statistical Characterization of Scanning Electron Microscopy Images. Industrial \& Engineering Chemistry Research, 56(34), pp.9496-9512.

Dutta, T. et al., 2013. Experiment and simulation of multifractal growth of crystalline $\mathrm{NaCl}$ aggregates in aqueous gelatin medium. Colloids and Surfaces A: Physicochemical and Engineering Aspects, 432, pp.127-131.

Edwards, C. et al., 2015. Measuring the nonuniform evaporation dynamics of sprayed sessile microdroplets with quantitative phase imaging. Langmuir, 31(40), pp.11020-11032.

Fan, J.A. et al., 2010. Self-assembled plasmonic nanoparticle clusters. Science, 328(5982), pp.11351138.

Feder, J., 2013. Fractals, Springer Science \& Business Media.

Field, A., Miles, J. \& Field, Z., 2012. Discovering statistics using R, Sage publications.

Gibbons, J.D. \& Chakraborti, S., 2010. Nonparametric Statistical Inference, CRC Press.

Haracz, S. et al., 2015. Magnetic behaviour of non-interacting colloidal iron oxide nanoparticles in physiological solutions. Crystal Research and Technology, 50(9-10), pp.791-796.

Haralick, R.M., Shanmugam, K. \& others, 1973. Textural features for image classification. IEEE Transactions on systems, man, and cybernetics, (6), pp.610-621.

He, L., Wang, G. \& Zhang, Q., 2003. An alternative paclitaxel microemulsion formulation: hypersensitivity evaluation and pharmacokinetic profile. International Journal of Pharmaceutics, 250(1), pp.45-50.

He, R. et al., 2014. Facile synthesis of pentacle gold-copper alloy nanocrystals and their plasmonic and catalytic properties. Nature Communications, 5, p.4327.

Hu, H. \& Larson, R.G., 2006. Marangoni effect reverses coffee-ring depositions. The Journal of Physical Chemistry B, 110(14), pp.7090-7094.

Huang, X. et al., 2017. Resveratrol encapsulation in core-shell biopolymer nanoparticles: Impact on antioxidant and anticancer activities. Food Hydrocolloids, 64, pp.157-165.

Jiao, Y. et al., 2015. Fabrication of three-dimensionally interconnected nanoparticle superlattices and their lithium-ion storage properties. Nature Communications, 6, p.6420. 
Jing, J. et al., 1998. Automated high resolution optical mapping using arrayed, fluid-fixed DNA molecules. Proceedings of the National Academy of Sciences, 95(14), pp.8046-8051.

Khatun, T., Dutta, T. \& Tarafdar, S., 2013. Crack formation under an electric field in droplets of laponite gel: Memory effect and scaling relations. Langmuir, 29(50), pp.15535-15542.

Layani, M. et al., 2009. Transparent conductive coatings by printing coffee ring arrays obtained at room temperature. ACS Nano, 3(11), pp.3537-3542.

Lewandowski, W. et al., 2015. Dynamically self-assembled silver nanoparticles as a thermally tunable metamaterial. Nature Communications, 6, p.6590.

Loeb, J. \& Loeb, R.F., 1921. The influence of electrolytes on the solution and precipitation of casein and gelatin. The Journal of General Physiology, 4(2), pp.187-211.

Maity, D. et al., 2011. In situ synthesis, characterization, and antimicrobial activity of silver nanoparticles using water soluble polymer. Journal of Applied Polymer Science, 122(4), pp.21892196.

Mal, D. et al., 2006. Fractal crack patterns in Laponite films and their scaling behavior. Fractals, 14(04), pp.283-288.

Nakanishi, H. et al., 2009. Photoconductance and inverse photoconductance in films of functionalized metal nanoparticles. Nature, 460(7253), p.371.

Otsu, N., 1979. A threshold selection method from gray-level histograms. IEEE transactions on systems, man, and cybernetics, 9(1), pp.62-66.

Pappas, T.N., 1992. An adaptive clustering algorithm for image segmentation. IEEE Transactions on signal processing, 40(4), pp.901-914.

Park, J. \& Moon, J., 2006. Control of colloidal particle deposit patterns within picoliter droplets ejected by ink-jet printing. Langmuir, 22(8), pp.3506-3513.

Pradhan, T.K. \& Panigrahi, P.K., 2015. Deposition pattern of interacting droplets. Colloids and Surfaces A: Physicochemical and Engineering Aspects, 482, pp.562-567.

Pradhan, T.K. \& Panigrahi, P.K., 2016. Influence of an adjacent droplet on fluid convection inside an evaporating droplet of binary mixture. Colloids and Surfaces A: Physicochemical and Engineering Aspects, 500, pp.154-165.

Roy, A. et al., 1999. Discrete scale invariance in viscous fingering patterns. The European Physical Journal B-Condensed Matter and Complex Systems, 12(1), pp.1-3.

Roy, B. et al., 2015. Multi-scale patterns formed by sodium sulphate in a drying droplet of gelatin. Applied Surface Science, 357, pp.1000-1006.

Rozin, M.J. et al., 2015. Colloidal metasurfaces displaying near-ideal and tunable light absorbance in the infrared. Nature Communications, 6, p.7325.

Sefiane, K., 2014. Patterns from drying drops. Advances in Colloid and Interface Science, 206, pp.372-381. 
Shahidzadeh-Bonn, N. et al., 2008. Salt crystallization during evaporation: impact of interfacial properties. Langmuir, 24(16), pp.8599-8605.

Sinha, S. \& Tarafdar, S., 2009. Viscous fingering patterns and evolution of their fractal dimension. Industrial \& Engineering Chemistry Research, 48(19), pp.8837-8841.

Tamas, V., 1992. Fractal growth phenomena, World Scientific.

Tao, A., Sinsermsuksakul, P. \& Yang, P., 2007. Tunable plasmonic lattices of silver nanocrystals. Nature Nanotechnology, 2(7), p.435.

Tarafdar, S. \& Sinha, S., 2008. Crack formation in drying laponite. Industrial \& Engineering Chemistry Research, 47(17), pp.6459-6464.

Tarasevich, Y.Y., Vodolazskaya, I.V. \& Sakharova, L.V., 2016. Mathematical modeling of pattern formation caused by drying of colloidal film under a mask. European Physical Journal E-Soft Matter, 39(2).

Theodoridis, S. et al., 2010. Introduction to pattern recognition: a matlab approach, Academic Press.

Wang, Q.-Q. et al., 2007. Highly efficient avalanche multiphoton luminescence from coupled Au nanowires in the visible region. Nano Letters, 7(3), pp.723-728.

Wong, T.-S. et al., 2011. Nanochromatography driven by the coffee ring effect. Analytical Chemistry, 83(6), pp.1871-1873.

Wong, T.W., 2011. Alginate graft copolymers and alginate-co-excipient physical mixture in oral drug delivery. Journal of Pharmacy and Pharmacology, 63(12), pp.1497-1512.

Yakhno, T., 2008. Salt-induced protein phase transitions in drying drops. Journal of Colloid and Interface Science, 318(2), pp.225-230.

Yoon, B. et al., 2014. Hydrogen-bonded structure and mechanical chiral response of a silver nanoparticle superlattice. Nature Materials, 13(8), p.807.

Zang, D. et al., 2019. Evaporation of a Droplet: From physics to applications. Physics Reports.

Zhang, Z., Zhang, R. \& McClements, D.J., 2017. Lactase (\$beta \$-galactosidase) encapsulation in hydrogel beads with controlled internal $\mathrm{pH}$ microenvironments: Impact of bead characteristics on enzyme activity. Food Hydrocolloids, 67, pp.85-93.

Zijlstra, P., Chon, J.W. \& Gu, M., 2009. Five-dimensional optical recording mediated by surface plasmons in gold nanorods. Nature, 459(7245), p.410. 